\title{
Asymmetric Truncated Hankel Operators: Rank One, Matrix Representation
}

\author{
Firdaws Rahmani $\mathbb{D}^{1},{ }^{1}$ Yufeng $L u\left(\mathbb{D},{ }^{1}\right.$ and $\operatorname{Ran} \mathrm{Li}\left(\mathbb{D}^{2}\right.$ \\ ${ }^{1}$ School of Mathematical Sciences, Dalian University of Technology, Dalian, China \\ ${ }^{2}$ School of Mathematics, Liaoning Normal University, Dalian, China \\ Correspondence should be addressed to Ran Li; liranmika@163.com
}

Received 9 April 2021; Accepted 14 August 2021; Published 6 September 2021

Academic Editor: Kehe Zhu

Copyright (c) 2021 Firdaws Rahmani et al. This is an open access article distributed under the Creative Commons Attribution License, which permits unrestricted use, distribution, and reproduction in any medium, provided the original work is properly cited.

\begin{abstract}
Asymmetric truncated Hankel operators are the natural generalization of truncated Hankel operators. In this paper, we determine all rank one operators of this class. We explore these operators on finite-dimensional model spaces, in particular, their matrix representation. We also give their matrix representation and the one for asymmetric truncated Toeplitz operators in the case of model spaces associated to interpolating Blaschke products.
\end{abstract}

\section{Introduction}

Let $H^{2}$ be the standard Hardy space of the unit disc $\mathbb{D}$ identified with the subspace of the boundary functions of its functions in $L^{2}(\mathbb{T})$.

A function in $H^{\infty}(\mathbb{D})$ is inner if it is unimodular on the unit circle $\mathbb{T}$. To each inner function $\alpha$ we associate a model space, $K_{\alpha}=H^{2} \ominus \alpha H^{2}$. The model space is a reproducing kernel Hilbert space with reproducing kernel, $k_{\lambda}^{\alpha}(z)=(1-$ $\overline{\alpha(\lambda)} \alpha(z)) /(1-\bar{\lambda} z)$, for $z, \lambda \in \mathbb{D}$.

The inner function $\alpha$ has an angular derivative in the sense of Carathéodory (ADC) at a point $\eta \in \mathbb{T}$ if and only if every $f$ in $K_{\alpha}$ has nontangential limit at $\eta$. In particular, $k_{\eta}^{\alpha} \in K_{\alpha}$.

If $\alpha$ is an inner function, then $\alpha^{\#}=\overline{\alpha(\bar{z})}$ is also an inner function and the associated model space is noted $K_{\alpha^{*}}$.

To each model space, we associate a natural conjugation $C_{\alpha}$ such that $C_{\alpha} K_{\alpha}=K_{\alpha}$, given by $C_{\alpha} f(z)=\alpha \overline{z f(z)}$, for $z \in \mathbb{T}$. The image of the kernel function for $C_{\alpha}$, called conjugate kernel function $\tilde{k}_{\lambda}^{\alpha}$, is given by $\tilde{k}_{\lambda}^{\alpha}(z)=(\alpha(z)-\alpha(\lambda)) /(z-\lambda)$, $z, \lambda \in \mathbb{D}$. If $\alpha$ has an ADC at $\eta \in \mathbb{T}$,

$\tilde{k}_{\eta}^{\alpha}(z)=\frac{\alpha(z)-\alpha(\eta)}{z-\eta}=\alpha(\eta) \bar{\eta} \frac{1-\overline{\alpha(\eta)} \alpha(z)}{1-\bar{\eta} z}=\alpha(\eta) \bar{\eta} k_{\eta}^{\alpha}(z)$
The model spaces can also be defined as the only invariant subspaces of the backward shift operator $S^{*}$ on $H^{2}$. Denote by $S_{\alpha}\left(S_{\alpha}^{*}\right)$ the compression of the shift operator (resp., backward shift) to the model space $K_{\alpha}$.

The only finite-dimensional model spaces are the one associated to finite Blaschke products, and its dimension is the same as the multiplicity of the associated Blaschke product. Since a finite Blaschke product is analytic on a neighborhood of the unit disc, it has an ADC everywhere on $\mathbb{T}$ and $k_{\eta}^{\alpha} \in K_{\alpha}$, for every $\eta \in \mathbb{T}$. In this case, let $m$ denote the multiplicity of a finite Blaschke product $\alpha$. If we arbitrarily choose a collection $\left\{\lambda_{i}, i=1, \cdots, m\right\}$ of distinct points in $\mathbb{D},\left\{k_{\lambda_{i}}^{\alpha}, i=1,2, . ., m\right\}$ forms a basis for $K_{\alpha}$.

In infinite-dimensional case the kernel functions $\left(k_{\lambda_{i}}^{\alpha}\right)_{i \geq 1}$ form a Riesz basis if and only if $\alpha$ is an interpolating Blaschke product or equivalently, $\left(\lambda_{i}\right)_{i \geq 1}$ is a uniformly separated Blaschke sequence that satisfies $\inf _{k \geq 1} \prod_{k \geq, k \neq n} \mid\left(\lambda_{k}-\lambda_{n}\right) /(1-$ $\left.\overline{\lambda_{k}} \lambda_{n}\right) \mid>0$.

Asymmetric truncated Toeplitz and Hankel operators were first introduced in $[1,2]$, respectively. For $\varphi \in L^{2}(\mathbb{T})$, let $\alpha$ and $\beta$ be two inner functions, the asymmetric truncated Toeplitz operator $A_{\varphi}^{\alpha, \beta}$ and the asymmetric truncated Hankel operator $B_{\varphi}^{\alpha, \beta}$ are defined on $K_{\alpha}^{\infty}=K_{\alpha} \cap H^{\infty}$ by 


$$
\begin{gathered}
A_{\varphi}^{\alpha, \beta}: K_{\alpha}^{\infty} \longrightarrow K_{\beta}, \\
f \mapsto A_{\varphi}^{\alpha, \beta}(f)=P_{\beta}(\varphi f), \\
B_{\varphi}^{\alpha, \beta}: K_{\alpha}^{\infty} \longrightarrow K_{\beta}, \\
f \mapsto B_{\varphi}^{\alpha, \beta}(f)=P_{\beta} J(I-P)(\varphi f),
\end{gathered}
$$

where $P$ and $P_{\beta}$ denote the orthogonal projection on $H^{2}$ and $K_{\beta}$, respectively, and $J$ is the flip operator from $H^{2}$ onto $\bar{H}_{0}^{2}=\overline{z H^{2}}$ defined on $\mathbb{T}$ by $J f(z)=\bar{z} f(\bar{z})$. We denote the set of bounded asymmetric truncated Toeplitz operators (ATTO) (bounded asymmetric truncated Hankel operators $(\mathrm{ATHO}))$ by $\mathfrak{T}(\alpha, \beta)(\mathfrak{S}(\alpha, \beta))$.

In [3], Sarason introduced truncated Toeplitz operators and showed that all rank one TTOs are of the form $c k_{\lambda}^{\alpha} \otimes$ $\tilde{k}_{\lambda}^{\alpha}$ or $c \tilde{k}_{\lambda}^{\alpha} \otimes k_{\lambda}^{\alpha}$, where $c$ in $\mathbb{C}$ and $\lambda$ in $\mathbb{D}$ or in $\mathbb{T}$ such that $\alpha$ has an ADC at $\lambda$. Similar results were obtained in [4] for truncated Hankel operators $\left(\tilde{k}_{\bar{\lambda}}^{\alpha} \otimes \tilde{k}_{\lambda}^{\alpha}, k_{\bar{\lambda}}^{\alpha} \otimes k_{\lambda}^{\alpha}\right)$. Surprisingly, this is not always true in the case of asymmetric truncated Toeplitz operators.

Theorem 1 (see $[5,6])$. Let $\alpha$ and $\beta$ be two inner functions.

(1) Let $\omega$ in $\mathbb{D}$ or $\omega$ in $\mathbb{T}$ such that $\alpha$ and $\beta$ has an $A D C$ at $\omega$

$$
k_{\omega}^{\beta} \otimes \tilde{k}_{\omega}^{\alpha}=A_{\frac{\alpha}{z \omega}}^{\alpha, \beta} \in \mathfrak{T}(\alpha, \beta) \text { and } \tilde{k}_{\omega}^{\beta} \otimes k_{\omega}^{\alpha}=A_{\frac{\beta}{z-\omega}}^{\alpha, \beta} \in \mathfrak{T}(\alpha, \beta) .
$$

(2) The only rank one asymmetric truncated Toeplitz operators in $\mathfrak{T}(\alpha, \beta)$ are nonzero scalar multiple of $k_{\omega}^{\beta} \otimes \tilde{k}_{\omega}^{\alpha}$ and $\tilde{k}_{\omega}^{\beta} \otimes k_{\omega}^{\alpha}$ where $\omega \in \mathbb{D}$ or $\omega \in \mathbb{T}$ such that $\alpha$ and $\beta$ have an ADC at $\omega$ if and only if $\{m n \leq 2\}$ or $\{m>1$ and $n>1\}$, where $m, n \in \mathbb{N} \cup\{+\infty\}$ are the dimensions of $K_{\alpha}$ and $K_{\beta}$, respectively

In [7], Cima et al. raised the question of which linear transformations on finite-dimensional model spaces are truncated Toeplitz operators, and they proved that the matrix representation of TTOs with respect to kernel basis (conjugate kernel basis, Clark and modified Clark bases) is entirely determined by the entries of the main diagonal and first row. In $[8,9]$, Lanucha obtained similar results for TTOs acting on model spaces associated to interpolating Blaschke products and for THOs. In [6], Jurasik and Lanucha generalized these results to asymmetric truncated Toeplitz operators on finite-dimensional model spaces.

This paper determines all rank one asymmetric truncated Hankel operators and generalizes the results about matrix representation to ATHOs and ATTOs on special model spaces. In Section 2, we cite some results from [3, 10-12]. We precise all rank one ATHOs in Section 3. In Section 4 , we explore the ATHOs on finite-dimensional spaces, we calculate the dimension of $\mathfrak{S}(\alpha, \beta)$ and exhibit a basis for it, and we give the matrix representation of ATHOs in finite dimensional model spaces associated to Blaschke products each with distinct zeros. Section 5 is dedicated to the matrix representation of ATHOs and ATTOS on special infinitedimensional model spaces, in particular, we study the action of the unitary operator $V_{\xi, c}=\sqrt{\tau_{\xi, c}^{\prime}} f \circ \tau_{\xi, c}$, where $\tau_{\xi, c}(z)$ is disc automorphism.

\section{Preliminaries}

Like for truncated Toeplitz and Hankel operators, the symbol of an ATTO and an ATHO is not unique, in fact, we have the following theorem.

Theorem 2 (see $[10,12])$. Let $\alpha$ and $\beta$ be inner functions. For $\varphi \in L^{2}(\mathbb{T})$, we have $A_{\varphi}^{\alpha, \beta}=0$ if and only if $\varphi \in \alpha \bar{H}^{2}+\beta H^{2}$ and $B_{\varphi}^{\alpha, \beta}=0$ if and only if $\varphi \in H^{2}+\overline{\alpha \beta^{\#} H^{2}}$. Where $\beta^{\#}(z)=\overline{\beta(\bar{z})}$.

We will use the following technical lemma.

Lemma 3 (see [3]). Let $\alpha$ be inner, for $\lambda \in \mathbb{D}$, we have

$$
S_{\alpha}^{*} k_{\lambda}^{\alpha}=\bar{\lambda} k_{\lambda}^{\alpha}-\overline{\alpha(\lambda)} \widetilde{k_{0}^{\alpha}}, \quad \text { and } \quad S_{\alpha} \tilde{k}_{\lambda}^{\alpha}=\lambda \tilde{k}_{\lambda}^{\alpha}-\alpha(\lambda) k_{0}^{\alpha} .
$$

For $\lambda \in \mathbb{D} \backslash\{0\}$, we have

$$
S_{\alpha}^{*} \widetilde{k_{\lambda}^{\alpha}}=\frac{1}{\bar{\lambda}} \widetilde{k_{\lambda}^{\alpha}}-\frac{1}{\lambda} \widetilde{k_{0}^{\alpha}}
$$

Clark, in [11], proved that the only unitary rank one perturbations of the compressed shift operator are

$$
U_{\lambda}^{\alpha}=S_{\alpha}+\frac{\alpha(0)+\lambda}{1-|\alpha(0)|^{2}} k_{0}^{\alpha} \otimes \widetilde{k_{0}^{\alpha}}, \quad \text { for } \quad \lambda \in \mathbb{T}
$$

and that the point spectrum of $U_{\lambda}^{\alpha}$ is the set of the solutions of the equation $\alpha(\eta)=\alpha(0)+\lambda / 1+\alpha \overline{(0)} \lambda$ at which $\alpha$ has an $A D C$, denote them by $\eta_{m}$. The corresponding eigenvectors are the normalized boundary kernels $v_{\eta_{m}}^{\alpha}:=\left\|k_{\eta_{m}}^{\alpha}\right\|^{-1} k_{\eta_{m}}^{\alpha}$ corresponding to the points $\eta_{m}$. Whenever the point spectrum of $U_{\lambda}^{\alpha}$ is pure, the family of eigenvectors forms a basis for $K_{\alpha}$ called the Clark basis.

The modified Clark basis $\left(e_{\eta_{m}}^{\alpha}\right)_{m}$ satisfies $C_{\alpha} e_{\eta_{m}}^{\alpha}=e_{\eta_{m}}^{\alpha}$ and is given by $e_{\eta_{m}}^{\alpha}=\omega_{m}^{\alpha} v_{\eta_{m}}^{\alpha}$, where $\omega_{m}^{\alpha}=\exp \left(-i\left(\arg \eta_{m}-\arg \lambda\right)\right)$.

One of the most important results about TTOs and THOs is their characterization in terms of compressed shift operator and operators of rank at most 2. Recently, the authors in [10] proved similar characterizations for both ATTO and ATHO.

Theorem 4 (see [10]). Let $A$ be a bounded linear operator from $K_{\alpha}$ to $K_{\beta}$. Then 
(1) $A \in \mathfrak{I}(\alpha, \beta)$ if and only if

$$
A-S_{\beta} A S_{\alpha}^{*}=\psi \otimes k_{0}^{\alpha}+k_{0}^{\beta} \otimes \chi,
$$

for some $\chi \in K_{\alpha}, \psi \in K_{\beta}$ if and only if

$$
A-U_{\lambda_{\beta}}^{\beta} A\left(U_{\lambda_{\alpha}}^{\beta}\right)^{*}=\psi \otimes k_{0}^{\alpha}+k_{0}^{\beta} \otimes \chi,
$$

for some $\chi \in K_{\alpha}, \psi \in K_{\beta}$ and some $\lambda_{\alpha}, \lambda_{\beta}$ in $\mathbb{T}$.

(2) $B \in \mathfrak{H}(\alpha, \beta)$ if and only if

$$
B-S_{\beta}^{*} B S_{\alpha}^{*}=\psi \otimes k_{0}^{\alpha}+\tilde{k}_{0}^{\beta} \otimes \chi,
$$

for some $\chi \in K_{\alpha}, \psi \in K_{\beta}$ if and only if

$$
B-\left(U_{\lambda_{\beta}}^{\beta}\right)^{*} B\left(U_{\lambda_{\alpha}}^{\alpha}\right)^{*}=\psi \otimes k_{0}^{\alpha}+\tilde{k}_{0}^{\beta} \otimes \chi,
$$

for some $\chi \in K_{\alpha}, \psi \in K_{\beta}$ and some $\lambda_{\alpha}, \lambda_{\beta}$ in $\mathbb{T}$.

Where $U_{\lambda_{\alpha}}^{\alpha}, U_{\lambda_{\beta}}^{\beta}$ are Clark operators (9).

In the same paper [10], we also have the following theorem.

Theorem 5 (see [10]). Let $\alpha$ and $\beta$ be two inner functions and $\varphi \in L^{2}(\mathbb{T})$. Then

(1) $A \in \mathfrak{T}(\alpha, \beta)$ if and only if $C_{\beta} A C_{\alpha} \in \mathfrak{T}(\alpha, \beta)$. In addition, $C_{\beta} A_{\varphi}^{\alpha, \beta} C_{\alpha}=A_{\bar{\alpha} \beta \bar{\varphi}}^{\alpha, \beta}$

(2) $B \in \mathfrak{H}(\alpha, \beta)$ if and only if $C_{\beta} B C_{\alpha} \in \mathfrak{H}(\alpha, \beta)$. In addition, $C_{\beta} B_{\varphi}^{\alpha, \beta} C_{\alpha}=B^{\alpha, \beta} \beta^{\#} \varphi$

(3) $C_{\beta} A_{\varphi}^{\alpha, \beta} J^{\#}=B_{\beta^{\#} \varphi^{\#}}^{\alpha^{\#}, \beta}$, where $J^{\#}$ is a conjugation on $L^{2}$ defined by $J^{\#} f=f(\bar{z})$

\section{Asymmetric Truncated Hankel Operators of Rank One}

In this section, we describe all rank one asymmetric truncated Hankel operators through the results for asymmetric truncated Toeplitz operators. In what follows, let $\alpha$ and $\beta$ denote two arbitrary inner functions.

The following proposition gives some rank one asymmetric truncated Hankel operators.

Proposition 6. We have

(1) For every $\lambda \in \mathbb{D}$, the operators $k_{\bar{\lambda}}^{\beta} \otimes k_{\lambda}^{\alpha}=B_{1 / z-\lambda}^{\alpha, \beta}$ and $\tilde{k}_{\bar{\lambda}}^{\beta} \otimes \tilde{k}_{\lambda}^{\alpha}=B_{\alpha \beta^{\#} / z \bar{\lambda}}^{\alpha, \beta}$ belong to $\mathfrak{W}(\alpha, \beta)$
(2) If $\alpha$ and $\beta$ have an $A D C$ at $\eta$ and $\bar{\eta}$, respectively, the operators $\tilde{k}_{\bar{\eta}}^{\beta} \otimes \tilde{k}_{\eta}^{\alpha}=B_{\alpha \beta^{\alpha, \beta}{ }_{I z-\eta}}^{\alpha}=B_{\tilde{k}_{\eta}^{\alpha, \beta}}^{\alpha, \beta}$ and $k_{\bar{\eta}}^{\beta} \otimes k_{\eta}^{\alpha}=$

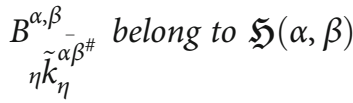

Proof. From Theorem 5, we have

$$
C_{\beta} A_{\varphi}^{\alpha, \beta} J^{\#}=B_{\beta^{\#} \varphi^{\#}}^{\alpha^{\#}, \beta}
$$

or

$$
C_{\beta} A_{\varphi}^{\alpha^{\#}, \beta} J^{\#}=B_{\beta^{\#} \varphi^{\#}}^{\alpha, \beta} .
$$

Choose an arbitrary $\lambda \in \mathbb{D}$ or $\lambda \in \mathbb{T}$ such that $\alpha^{\#}$ and $\beta$ have an $\mathrm{ADC}$ at $\lambda$, by Theorem 1, we have

$$
\begin{aligned}
& C_{\beta}\left(k_{\lambda}^{\beta} \otimes \tilde{k}_{\lambda}^{\alpha^{\#}}\right) J^{\#}=C_{\beta} A_{\frac{\alpha^{\#}}{z \lambda}}^{\alpha^{\#}, \beta} J^{\#}=B^{\alpha, \beta} \frac{\alpha \bar{\beta}^{\#}}{\bar{z}-\lambda}=\tilde{k}_{\lambda}^{\beta} \otimes \tilde{k}_{\bar{\lambda}}^{\alpha}, \\
& C_{\beta}\left(\tilde{k}_{\lambda}^{\beta} \otimes k_{\lambda}^{\alpha^{\#}}\right) J^{\#}=C_{\beta} A_{\frac{\beta}{z-\lambda}}^{\alpha^{\#}, \beta} J^{\#}=B_{\frac{1}{z-\lambda}}^{\alpha, \beta}=k_{\lambda}^{\beta} \otimes k_{\bar{\lambda}}^{\alpha} .
\end{aligned}
$$

For $\lambda \in \mathbb{D}$, the operators $\tilde{k}_{\lambda}^{\beta} \otimes \tilde{k}_{\bar{\lambda}}^{\alpha}$ and $k_{\lambda}^{\beta} \otimes k_{\bar{\lambda}}^{\alpha}$ belong to $\mathfrak{S}(\alpha, \beta)$. This is also true for $\lambda \in \mathbb{T}$, since $\alpha^{\#}$ has an ADC at $\lambda$, if and only if $\alpha$ has an ADC at $\bar{\lambda}$.

Now, we give the main theorem of this section.

Theorem 7. All rank one operators in $\mathfrak{H}(\alpha, \beta)$ are the nonzero scalar multiples of $k_{\bar{\lambda}}^{\beta} \otimes k_{\lambda}^{\alpha}$ and $\tilde{k}_{\bar{\lambda}}^{\beta} \otimes \tilde{k}_{\lambda}^{\alpha}$, where $\lambda \in \mathbb{D}$ or $\lambda \in \mathbb{T}$ such that $\alpha$ and $\beta$ have an $A D C$ at $\lambda$ and $\bar{\lambda}$, respectively, if and only if $\{m n \leq 2\}$ or $\{m>1$ and $n>1\}$, where $m, n \in$ $\mathbb{N} \cup\{+\infty\}$ are the dimensions of $K_{\alpha}$ and $K_{\beta}$, respectively.

Proof. Every rank one operator in $\mathfrak{H}(\alpha, \beta)$ is of the form $f$ $\otimes g$ for $f \in K_{\beta}$ and $g \in K_{\alpha}$. Since $\mathfrak{H}(\alpha, \beta)=C_{\beta} \mathfrak{I}\left(\alpha^{\#}, \beta\right) J^{\#}$ ([10]), we can find $f^{\prime} \in K_{\beta}$ and $g^{\prime} \in K_{\alpha^{\#}}$ such that $f \otimes g=$ $C_{\beta} f^{\prime} \otimes g^{\prime} J^{\#}$. For $f^{\prime} \otimes g^{\prime}$ is rank one in $\mathfrak{T}\left(\alpha^{\#}, \beta\right)$, by Theorem 1 , there exist $c \in \mathbb{C}$ and $\lambda \in \mathbb{D}$ or $\lambda \in \mathbb{T}\left(\alpha^{\#}, \beta\right.$ have an $\mathrm{ADC}$ at $\lambda$ ) such that $f^{\prime} \otimes g^{\prime}=c k_{\lambda}^{\beta} \otimes \tilde{k}_{\lambda}^{\alpha^{\#}}$ or $f^{\prime}$ $\otimes g^{\prime}=c \tilde{k}_{\lambda}^{\beta} \otimes k_{\lambda}^{\alpha^{\#}}$, if and only if $\{m n \leq 2\}$ or $\{m>1$ and $n>1\}$. Finally, $f \otimes g=c C_{\beta}\left(k_{\lambda}^{\beta} \otimes \tilde{k}_{\lambda}^{\alpha^{\#}}\right) J^{\#}=c \tilde{k}_{\lambda}^{\beta} \otimes \tilde{k}_{\bar{\lambda}}^{\alpha}$ or $f \otimes$ $g=c C_{\beta}\left(\tilde{k}_{\lambda}^{\beta} \otimes k_{\lambda}^{\alpha^{\#}}\right) J^{\#}=c k_{\lambda}^{\beta} \otimes k_{\bar{\lambda}}^{\alpha}$ if and only if $\{m n \leq 2\}$ or $\{m>1$ and $n>1\}$. 


\section{Asymmetric Truncated Hankel Operators in Finite-Dimensional Model Spaces}

In this section, we suppose that both $\alpha$ and $\beta$ are finite Blaschke products of respective multiplicities $m$ and $n$.

\subsection{Dimension and Basis of $\mathfrak{H}(\alpha, \beta)$}

Theorem 8. Let $K_{\alpha}$ and $K_{\beta}$ have dimensions $m$ and $n$, respectively, then the dimension of $\mathfrak{H}(\alpha, \beta)$ equals $m+n-1$.

Proof. Using Theorem 2, we can write

$$
\mathfrak{H}(\alpha, \beta)=\left\{B_{\bar{\chi}}^{\alpha, \beta}: \chi \in K_{\alpha \beta^{\#}}\right\}
$$

where $\operatorname{dim} K_{\alpha \beta^{\#}}=m+n$. Since the constants are in $K_{\alpha \beta^{\#}}$ and $B_{c}^{\alpha, \beta}=0$ for all $c \in \mathbb{C}$,

$$
\operatorname{dim} \mathfrak{S}(\alpha, \beta)=\operatorname{dim} K_{\alpha \beta^{\#}}-1=m+n-1 .
$$

Remark 9. From Theorem 4, $C_{\beta} \mathfrak{T}\left(\alpha^{\#}, \beta\right) J^{\#}=\mathfrak{H}(\alpha, \beta)$. Since $C_{\beta}$ and $J^{\#}$ preserve the dimensions, $\operatorname{dim} \mathfrak{S}(\alpha, \beta)=$ $\operatorname{dim} \mathfrak{I}\left(\alpha^{\#}, \beta\right)=m+n-1$.

Theorem 10. Let $\alpha, \beta$ be two finite Blaschke products of respective multiplicities $m$ and $n$. Then for any $m+n-1$ distinct points from $\overline{\mathbb{D}}$, denoted by $\left\{\lambda_{i}\right\}_{i=1}^{m+n-1}$,

(1) $\left\{k_{\bar{\lambda}_{i}}^{\beta} \otimes k_{\lambda_{i}}^{\alpha}, i=1, . ., m+n-1\right\}$ is a basis of $\mathfrak{H}(\alpha, \beta)$

(2) $\left\{\tilde{k}_{\bar{\lambda}_{i}}^{\beta} \otimes \tilde{k}_{\lambda_{i}}^{\alpha}, i=1, \ldots, m+n-1\right\}$ is also a basis of $\mathfrak{J}(\alpha, \beta)$

Proof. We only prove (1), the other case follows by application of the conjugations. From [6], a basis of $\mathfrak{I}\left(\alpha^{\#}, \beta\right)$ is $\left\{\tilde{k}_{\overline{\lambda_{i}}}^{\beta} \otimes k_{\bar{\lambda}_{i}}^{\alpha^{\#}}, i=1, \cdots, m+n-1\right\}$. Using the proof of Proposition 6, $\left\{C_{\beta} \tilde{k}_{\bar{\lambda}_{i}}^{\beta} \otimes k_{\bar{\lambda}_{i}}^{\alpha^{\#}} J^{\#}=k_{\bar{\lambda}_{i}}^{\beta} \otimes k_{\lambda_{i}}^{\alpha}, i=1, \cdots, m+n-1\right\}$ is a basis of $\mathfrak{S}(\alpha, \beta)$.

Remark 11. We can prove the result directly as in [6] for ATTOs.

4.2. Matrix Representation of ATHO on Finite-Dimensional Spaces. In this subsection, we give the matrix representation of an ATHO acting on two finite dimensional model spaces, with respect to kernel and conjugate kernel bases, Clark and modified Clark bases. In all this section, suppose that the inner function $\alpha$ has distinct zeros $\left(a_{i}\right)_{i=1}^{m}$ and $\beta$ has distinct zeros $\left(b_{j}\right)_{j=1}^{n}$.
4.2.1. Matrix Representation with respect to the Kernel Bases and Conjugate Kernel Bases. Choose $m+n-1$ points $\left\{\lambda_{i}\right\}_{i=1}^{m+n-1}$ in $\mathbb{D}$ distinct from $\left(\bar{b}_{j}\right)_{j=1}^{n}$, then $\left\{k_{\bar{\lambda}_{i}}^{\beta} \otimes k_{\lambda_{i}}^{\alpha}, i=1, .\right.$. , $m+n-1\}$ is a basis for $\mathfrak{H}(\alpha, \beta)$ (Theorem 10). Since the zeros of the considered inner functions are distinct, $\left\{k_{a_{j}}^{\alpha}, j=1, \cdots\right.$, $m\}$ and $\left\{k_{b_{i}}^{\beta}, i=1, \cdots, n\right\}$ are bases of $K_{\alpha}$ and $K_{\beta}$ (same for the conjugate kernel functions). We denote the matrix representation of a bounded operator $B$ with respect to the abovementioned kernel bases (conjugate kernel bases) by $\left(s_{i, j}\right)\left(\left(p_{i, j}\right)\right)$, where $1 \leq i \leq n$ and $1 \leq j \leq m$. For the asymmetric case, we also have (see [9]),

$$
s_{i, j}=\frac{1}{\beta^{\prime\left(b_{i}\right)}}\left\langle B k_{a_{j}}^{\alpha}, \tilde{k}_{b_{i}}^{\beta}\right\rangle \text {. }
$$

Theorem 12. Let $B$ be a bounded linear transformation from $K_{\alpha}$ to $K_{\beta}$.

$B \in \mathfrak{S}(\alpha, \beta)$ if and only if for $1 \leq i \leq n$ and $1 \leq j \leq m$,

$s_{i, j}=\frac{\bar{\beta}^{\prime}\left(b_{1}\right)\left(1-a_{j} \bar{b}_{1}\right) s_{1, j}-\bar{\beta}^{\prime}\left(b_{1}\right)\left(1-\bar{a}_{1} \bar{b}_{1}\right) s_{1,1}+\bar{\beta}^{\prime}\left(b_{i}\right)\left(1-\overline{a_{1} b_{i}}\right) s_{i, 1}}{\bar{\beta}^{\prime}\left(b_{i}\right)\left(1-\bar{a}_{j} b_{i}\right)}$.

Proof. Suppose that $B=B_{\varphi}^{\alpha, \beta} \in \mathfrak{S}(\alpha, \beta)$ for some $\varphi \in L^{2}$. We show that its matrix representation with respect to the kernel basis is of the form (21). From Theorem $10, B_{\varphi}^{\alpha, \beta}=\sum_{q=1}^{m+n-1}$

$d_{q} k_{\lambda_{q}}^{\beta} \otimes k_{\lambda_{q}}^{\alpha}$. We have

$$
B_{\varphi}^{\alpha, \beta} k_{a_{j}}^{\alpha}=\left(\sum_{q=1}^{m+n-1} d_{q} k_{\bar{\lambda}_{q}}^{\beta} \otimes k_{\lambda_{q}}^{\alpha}\right) k_{a_{j}}^{\alpha}=\sum_{q=1}^{m+n-1} d_{q} \frac{1}{1-\bar{a}_{j} \lambda_{q}} k_{\bar{\lambda}_{q}}^{\beta} .
$$

Now replace in (20), we have

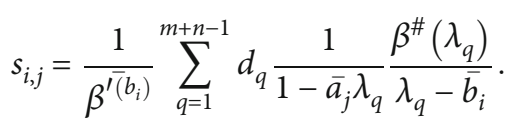

We can write

$$
\frac{1}{1-\bar{a}_{j} \lambda_{q}} \frac{1}{\lambda_{q}-\bar{b}_{i}}=\frac{1}{\left(1-\bar{a}_{j} \bar{b}_{i}\right)\left(1-\lambda_{q}\right)}\left(\frac{1-\bar{b}_{i}}{\lambda_{q}-\bar{b}_{i}}-\frac{1-\bar{a}_{j}}{1-\lambda_{q} \bar{a}_{j}}\right) .
$$

By adding and subtracting $1-\overline{b_{1}} / \lambda_{q}-\bar{b}_{1}$ and $1-\overline{a_{1}} / 1$ $-\lambda_{q} \overline{a_{1}}$, we find

$s_{i, j}=\frac{\bar{\beta}^{\prime}\left(b_{1}\right)\left(1-a_{j} \bar{b}_{1}\right) s_{1, j}-\bar{\beta}^{\prime}\left(b_{1}\right)\left(1-\bar{a}_{1} \bar{b}_{1}\right) s_{1,1}+\bar{\beta}^{\prime}\left(b_{i}\right)\left(1-\overline{a_{1} b_{i}}\right) s_{i, 1}}{\bar{\beta}^{\prime}\left(b_{i}\right)\left(1-\bar{a}_{j} b_{i}\right)}$ 
Conversely, suppose that the matrix representation of the bounded transformation $B$ satisfies (21). From the proof of the first implication, we know that the subspace of the matrices satisfying (21) is a subspace of $\mathfrak{S}(\alpha, \beta)$, and its dimension is obviously $m+n-1$. But $\operatorname{dim} \mathfrak{H}(\alpha, \beta)=m+n$ -1 , we have the equality.

The following theorem establishes the matrix representation with respect to conjugate kernel bases.

Theorem 13. Let $B$ be a bounded linear transformation from $K_{\alpha}$ to $K_{\beta} . B \in \mathfrak{H}(\alpha, \beta)$ if and only if for $1 \leq i \leq n$ and $1 \leq j \leq m$

$p_{i, j}=\frac{\beta^{\prime}\left(b_{1}\right)\left(1-a_{j} b_{1}\right) p_{1, j}-\beta^{\prime}\left(b_{1}\right)\left(1-a_{1} b_{1}\right) p_{1,1}+\beta^{\prime}\left(b_{i}\right)\left(1-a_{1} b_{i}\right) p_{i, 1}}{\beta^{\prime}\left(b_{i}\right)\left(1-a_{j} b_{i}\right)}$.

Proof. We have

$$
\begin{aligned}
p_{i, j} & =\frac{1}{\beta^{\prime}\left(b_{i}\right)}\left\langle B \tilde{k}_{a_{j}}^{\alpha}, k_{b_{i}}^{\beta}\right\rangle=\frac{1}{\beta^{\prime}\left(b_{i}\right)}\left\langle B C_{\alpha} k_{a_{j}}^{\alpha}, C_{\beta} \tilde{k}_{b_{i}}^{\beta}\right\rangle \\
= & = \\
= & \frac{1}{\beta^{\prime\left(b_{i}\right)}}\left\langle C_{\beta} B C_{\alpha} k_{a_{j}}^{\alpha}, \tilde{k}_{b_{i}}^{\beta}\right\rangle=\frac{1}{\beta^{\prime\left(b_{i}\right)}}\left\langle B^{\alpha, \beta} \beta^{\#} k^{\beta} k_{a_{j}}^{\alpha}, \tilde{k}_{b_{i}}^{\beta}\right\rangle .
\end{aligned}
$$

Therefore

$$
p_{i, j}=\overline{s_{i, j}}
$$

where $s_{i, j}$ is the entry in the ith row and jth column of the matrix representing $B_{\alpha \beta^{\#} \varphi}^{\alpha, \beta}$ with respect to the kernel bases, by Theorem 12, it satisfies (21) and we have

$$
\begin{aligned}
p_{i, j} & =\frac{\bar{\beta}^{\prime}\left(b_{1}\right)\left(1-a_{j} \bar{b}_{1}\right) s_{1, j}-\bar{\beta}^{\prime}\left(b_{1}\right)\left(1-a_{1} b_{1}\right) s_{1,1}+\bar{\beta}^{\prime}\left(b_{i}\right)\left(1-a_{1} b_{i}\right) s_{i, 1}}{\overline{\beta^{\prime}}\left(b_{i}\right)\left(1-a_{j} b_{i}\right)} \\
& =\frac{\beta^{\prime}\left(b_{1}\right)\left(1-a_{j} b_{1}\right) s_{1, j}^{-}-\beta^{\prime}\left(b_{1}\right)\left(1-a_{1} b_{1}\right) s_{1,1}^{-}+\beta^{\prime}\left(b_{i}\right)\left(1-a_{1} b_{i}\right) s_{i, 1}^{-}}{\beta^{\prime}\left(b_{i}\right)\left(1-a_{j} b_{i}\right)} \\
& =\frac{\beta^{\prime}\left(b_{1}\right)\left(1-a_{j} b_{1}\right) p_{1, j}-\beta^{\prime}\left(b_{1}\right)\left(1-a_{1} b_{1}\right) p_{1,1}+\beta^{\prime}\left(b_{i}\right)\left(1-a_{1} b_{i}\right) p_{i, 1}}{\beta^{\prime}\left(b_{i}\right)\left(1-a_{j} b_{i}\right)} .
\end{aligned}
$$

4.2.2. Matrix Representation with respect to Clark and Modified Clark Bases. Let $\alpha$ be $m$-finite Blaschke product and $\beta$ be $n$-finite Blaschke product. Choose arbitrary $\lambda_{1}, \lambda_{2}$ $\in \mathbb{T}$, then the following equations

$$
\alpha(\eta)=\frac{\alpha(0)+\lambda_{1}}{1+\alpha \overline{(0)} \lambda_{1}}:=\alpha_{\lambda_{1}} \text { and } \beta(\eta)=\frac{\beta(0)+\lambda_{2}}{1+\beta \overline{(0) \lambda_{2}}}:=\beta_{\lambda_{2}},
$$

have exactly $m$ and $n$ distinct solutions, respectively, denoted by $\left(\eta_{j}\right)_{j=1}^{m}$ and $\left(\zeta_{i}\right)_{i=1}^{n}$. The families of normalized eigenvectors $v_{\eta_{j}}^{\alpha}:=\left\|k_{\eta_{j}}^{\alpha}\right\|^{-1} k_{\eta_{j}}^{\alpha}$ and $v_{\zeta_{i}}^{\beta}:=\left\|k_{\zeta_{i}}^{\beta}\right\|^{-1} k_{\zeta_{i}}^{\beta}$ form orthonormal bases for $K_{\alpha}$ and $K_{\beta}$, respectively. We also have

$$
\left\|k_{\eta_{j}}^{\alpha}\right\|=\sqrt{\left|\alpha^{\prime}\left(\eta_{j}\right)\right|} \text { and }\left\|k_{\zeta_{i}}^{\beta}\right\|=\sqrt{\left|\beta^{\prime}\left(\zeta_{i}\right)\right|} \text {. }
$$

Reorder the sequences $\left(\eta_{j}\right)_{j=1}^{m}$ and $\left(\zeta_{i}\right)_{i=1}^{n}$ so that $l$ be the maximal integer such that $\bar{\eta}_{i}=\zeta_{i}$, for any $i \leq l$. $l=0$ when $\bar{\eta}_{i}=\zeta_{i}$ for every $i$. Denote by $\left(t_{i, j}\right)$, for $1 \leq i \leq n$ and $1 \leq j \leq m$ , the matrix representation of any bounded transformation from $K_{\alpha}$ into $K_{\beta}$ with respect to Clark bases, and by $\left(u_{i, j}\right)$, for $1 \leq i \leq n$ and $1 \leq j \leq m$, the matrix representation with respect to the modified Clark bases, we have

$$
u_{i, j}=\left\langle B e_{\eta_{j}}^{\alpha}, e_{\zeta_{i}}^{\beta}\right\rangle=\omega_{j}^{\alpha} \overline{\omega_{i}^{\beta}}\left\langle B v_{\eta_{j}}^{\alpha}, v_{\zeta_{i}}^{\beta}\right\rangle=\omega_{j}^{\alpha} \omega_{i}^{\beta} t_{i, j}
$$

Theorem 14. A bounded linear transformation $B$ from $K_{\alpha}$ to $K_{\beta}$ belongs to $\mathfrak{T}(\alpha, \beta)$ if and only if

(1) For $l \geq 1$ with $i \leq l$ and $i=j$

$$
\begin{aligned}
t_{i, j}= & \frac{\sqrt{\left|\beta^{\prime}\left(\zeta_{1}\right)\right|}\left(\bar{\eta}_{j}-\zeta_{1}\right)}{\sqrt{\left|\beta^{\prime}\left(\zeta_{i}\right)\right|}\left(\bar{\eta}_{j}-\zeta_{i}\right)} t_{1, j} \\
& +\frac{\sqrt{\left|\alpha^{\prime}\left(\eta_{i}\right)\right|} \sqrt{\left|\beta^{\prime}\left(\zeta_{1}\right)\right|}\left(\bar{\eta}_{j}-\zeta_{i}\right)}{\sqrt{\left|\alpha^{\prime}\left(\eta_{j}\right)\right|} \sqrt{\left|\beta^{\prime}\left(\zeta_{i}\right)\right|}\left(\bar{\eta}_{j}-\zeta_{i}\right)} t_{1, i} .
\end{aligned}
$$

For $l \geq 1$ with $i>l$,

$$
\begin{aligned}
& t_{i, j}=\frac{\sqrt{\left|\beta^{\prime}\left(\zeta_{1}\right)\right|}\left(\bar{\eta}_{j}-\zeta_{1}\right)}{\sqrt{\left|\beta^{\prime}\left(\zeta_{i}\right)\right|}\left(\bar{\eta}_{j}-\zeta_{i}\right)} t_{1, j}+\frac{\sqrt{\left|\alpha^{\prime}\left(\eta_{1}\right)\right|}\left(\bar{\eta}_{j}-\zeta_{i}\right)}{\sqrt{\left|\alpha^{\prime}\left(\eta_{j}\right)\right|}\left(\bar{\eta}_{j}-\zeta_{i}\right)} t_{i, 1} . \\
& \text { (2) For } l=0
\end{aligned}
$$

$$
\begin{aligned}
t_{i, j}= & \frac{\sqrt{\left|\beta^{\prime}\left(\zeta_{1}\right)\right|}\left(\bar{\eta}_{j}-\zeta_{1}\right)}{\sqrt{\left|\beta^{\prime}\left(\zeta_{i}\right)\right|}\left(\bar{\eta}_{j}-\zeta_{i}\right)} t_{1, j}-\frac{\sqrt{\left|\alpha^{\prime}\left(\eta_{1}\right)\right|} \sqrt{\left|\beta^{\prime}\left(\zeta_{1}\right)\right|}\left(\bar{\eta}_{1}-\zeta_{1}\right)}{\sqrt{\left|\alpha^{\prime}\left(\eta_{j}\right)\right|}} t_{1,1} \sqrt{\left|\beta^{\prime}\left(\zeta_{i}\right)\right|}\left(\bar{\eta}_{j}-\zeta_{i}\right) \\
& +\frac{\sqrt{\left|\alpha^{\prime}\left(\eta_{1}\right)\right|}\left(\bar{\eta}_{1}-\zeta_{i}\right)}{\sqrt{\left|\alpha^{\prime}\left(\eta_{j}\right)\right|}\left(\bar{\eta}_{j}-\zeta_{i}\right)} t_{i, 1} .
\end{aligned}
$$

Proof. We proceed as in the proof of Theorem 12. 
We deduce the following theorem from Theorem 14 and formula (32).

Theorem 15. A bounded linear transformation $B$ from $K_{\alpha}$ to $K_{\beta}$ belongs to $\mathfrak{H}(\alpha, \beta)$ if and only if for $1 \leq i \leq n$ and $1 \leq j \leq m$ , except that $i=j \leq l$.

(1) For $l \geq 1$ with $i \leq l$ and $i=j$

$$
\begin{aligned}
u_{i, j}= & \frac{\omega_{1}^{\beta}}{\omega_{i}^{\beta}} \frac{\sqrt{\left|\beta^{\prime}\left(\zeta_{1}\right)\right|}\left(\bar{\eta}_{j}-\zeta_{1}\right)}{\sqrt{\left|\beta^{\prime}\left(\zeta_{i}\right)\right|}\left(\bar{\eta}_{j}-\zeta_{i}\right)} u_{1, j} \\
& +\frac{\omega_{j}^{\alpha}}{\omega_{1}^{\alpha}} \frac{\sqrt{\left|\alpha^{\prime}\left(\eta_{i}\right)\right|} \sqrt{\left|\beta^{\prime}\left(\zeta_{1}\right)\right|}\left(\bar{\eta}_{j}-\zeta_{i}\right)}{\sqrt{\left|\alpha^{\prime}\left(\eta_{j}\right)\right|} \sqrt{\left|\beta^{\prime}\left(\zeta_{i}\right)\right|}\left(\bar{\eta}_{j}-\zeta_{i}\right)} u_{1, i} \cdot
\end{aligned}
$$

For $l \geq 1$ with $i>l$,

$$
\begin{aligned}
u_{i, j}= & \frac{\omega_{1}^{\beta}}{\omega_{i}^{\beta}} \frac{\sqrt{\left|\beta^{\prime}\left(\zeta_{1}\right)\right|}\left(\bar{\eta}_{j}-\zeta_{i}\right)}{\sqrt{\left|\beta^{\prime}\left(\zeta_{i}\right)\right|}\left(\bar{\eta}_{j}-\zeta_{i}\right)} u_{1, j} \\
& +\frac{\omega_{j}^{\alpha}}{\omega_{1}^{\alpha}} \frac{\sqrt{\left|\alpha^{\prime}\left(\eta_{1}\right)\right|}\left(\bar{\eta}_{j}-\zeta_{i}\right)}{\sqrt{\left|\alpha^{\prime}\left(\eta_{j}\right)\right|}\left(\bar{\eta}_{j}-\zeta_{i}\right)} u_{i, 1} .
\end{aligned}
$$

(2) For $l=0$

$$
\begin{aligned}
u_{i, j}= & \frac{\omega_{1}^{\beta}}{\omega_{i}^{\beta}} \frac{\sqrt{\left|\beta^{\prime}\left(\zeta_{1}\right)\right|}\left(\bar{\eta}_{j}-\zeta_{i}\right)}{\sqrt{\left|\beta^{\prime}\left(\zeta_{i}\right)\right|}\left(\bar{\eta}_{j}-\zeta_{i}\right)} u_{1, j} \\
& -\frac{\omega_{j}^{\alpha}}{\omega_{i}^{\beta}} \frac{\omega_{1}^{\beta}}{\omega_{1}^{\alpha}} \frac{\sqrt{\left|\alpha^{\prime}\left(\eta_{1}\right)\right|} \sqrt{\left|\beta^{\prime}\left(\zeta_{1}\right)\right|}\left(\bar{\eta}_{j}-\zeta_{i}\right)}{\sqrt{\left|\alpha^{\prime}\left(\eta_{j}\right)\right|}} u_{1,1} \sqrt{\left|\beta^{\prime}\left(\zeta_{i}\right)\right|}\left(\bar{\eta}_{j}-\zeta_{i}\right) \\
& +\frac{\omega_{j}^{\alpha}}{\omega_{1}^{\alpha}} \frac{\sqrt{\left|\alpha^{\prime}\left(\eta_{1}\right)\right|}\left(\bar{\eta}_{j}-\zeta_{i}\right)}{\sqrt{\left|\beta^{\prime}\left(\zeta_{i}\right)\right|}\left(\bar{\eta}_{j}-\zeta_{i}\right)} u_{i, 1} .
\end{aligned}
$$

\section{Matrix Representation in Infinite- Dimensional Case}

In all this section, unless mentioned, we will suppose that $\alpha$ and $\beta$ are two interpolating Blaschke sequences with respective zeros $\left(a_{m}\right)_{m \geq 1}$ and $\left(b_{n}\right)_{n \geq 1}$, then the kernel functions $\left(k_{a_{i}}^{\alpha}\right)_{i \geq 1}$ and conjugate kernel functions $\left(\tilde{k}_{a_{i}}^{\alpha}\right)_{i \geq 1}$ form two Riesz bases for $K_{\alpha}$ (same for $K_{\beta}$ ) (see $[8,9]$ ). For all sequence of complex numbers $\left(f_{m}\right)_{m \geq 1}$ such that $\sum_{m=1}^{\infty}\left|f_{m}\right|^{2}\left(1-\left|a_{m}\right|^{2}\right)$ $<\infty$, the unique solution $f$ in $K_{\alpha}$ of the interpolation problem $f\left(a_{m}\right)=f_{m}$ is given by

$$
f=\sum_{i=1}^{+\infty} \frac{f\left(a_{i}\right)}{\alpha^{\prime}\left(a_{i}\right)} \tilde{k}_{a_{i}}^{\alpha}=\sum_{i=1}^{+\infty} \frac{\tilde{f}\left(a_{i}\right)}{\alpha^{\prime\left(a_{i}\right)}} k_{a_{i}}^{\alpha}
$$

We will also keep the previous notations of the matrix representations of an operator with respect to the different bases $\left(s_{i, j}, p_{i, j}, t_{i, j}\right.$, and $\left.u_{i, j}\right)$.

5.1. Matrix Representation of ATHOs. Denote by $\left(a_{l_{k}}\right)_{k \geq 1}=$ $\left(\bar{b}_{l_{k}}\right)_{k \geq 1}$ the subsequence of common elements between $\left(a_{m}\right)_{m}$ and $\left(\bar{b}_{n}\right)_{n}$ ordered such that $a_{l_{k}}=\bar{b}_{l_{k}}$ and that $1=l_{1}$ $\epsilon\left(l_{k}\right)_{k \geq 1}$. We will prove that the above matrix representations are also true in the case of model spaces associated to interpolating Blaschke products.

Theorem 16. Let $B$ be a bounded linear transformation from $K_{\alpha}$ to $K_{\beta}$. Then, $B \in \mathfrak{S}(\alpha, \beta)$ if and only if for any $i, j$

$$
s_{i, j}=\frac{\bar{\beta}^{\prime}\left(b_{1}\right)\left(1-a_{j} \bar{b}_{1}\right) s_{1, j}-\bar{\beta}^{\prime}\left(b_{1}\right)\left(1-a_{1} \bar{b}_{1}\right) s_{1,1}+\bar{\beta}^{\prime}\left(b_{i}\right)\left(1-\bar{a}_{1} b_{i}\right) s_{i, 1}}{\bar{\beta}^{\prime}\left(b_{i}\right)\left(1-a_{j} b_{i}\right)} .
$$

Proof. Suppose that $B=B_{\varphi}^{\alpha, \beta}$ and we will prove that it satisfies (40). As in the proof of Theorem 8 , for any $\varphi$ in $L^{2}(\mathbb{T})$, there is a $\phi \in K_{\alpha \beta^{\#}}$ such that $B_{\varphi}^{\alpha, \beta}=B_{\bar{\phi}}^{\alpha, \beta}$, where $K_{\alpha \beta^{\#}}=K_{\alpha} \oplus \alpha K_{\beta^{\#}}$. Then, there are $\chi \in K_{\alpha}$ and $\psi \in K_{\beta^{\#}}$ such that $B_{\varphi}^{\alpha, \beta}=B_{\bar{\chi}}^{\alpha, \beta}+$ $B_{\alpha \bar{\psi}}^{\alpha, \beta}$. Let $\beta^{\#}$ be an interpolating Blaschke product with zeros $\left(\bar{b}_{m}^{-}\right)_{m \geq 1}$. For the bases $\left(k_{a_{m}}^{\alpha}\right)_{m \geq 1}$ and $\left(\tilde{k}_{b_{n}}^{\beta^{\#}}\right)_{n \geq 1}$, we have

$$
\chi=\sum_{m=1}^{\infty} \overline{c_{m}} k_{a_{m}}^{\alpha} \text { and } \psi=\sum_{n=1}^{\infty} \overline{d_{n}} \tilde{k}_{\bar{b}_{n}^{-}}^{\beta^{\#}}
$$

Replacing in $B_{\varphi}^{\alpha, \beta}$,

$$
B_{\varphi}^{\alpha, \beta}=\sum_{m=1}^{\infty} c_{m} B_{k_{a_{m}}^{\alpha, \beta}}^{\alpha,}+\sum_{n=1}^{\infty} d_{n} B^{\alpha, \beta}=
$$

By (6), we have

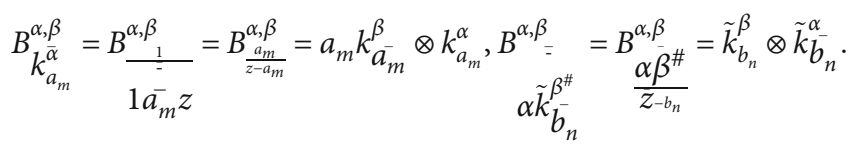

So

$$
B_{\varphi}^{\alpha, \beta}=\sum_{m=1}^{\infty} c_{m} a_{m} k_{a_{m}}^{\beta} \otimes k_{a_{m}}^{\alpha}+\sum_{n=1}^{\infty} \tilde{k}_{b_{n}}^{\beta} \otimes \tilde{k}_{b_{n}^{-}}^{\alpha}
$$


Replacing in (20), we get

$s_{i, j}=\frac{1}{\beta^{\prime}\left(b_{i}\right)}\left(\sum_{m=1}^{\infty} \frac{c_{m} a_{m}}{1-\bar{a}_{j} a_{m}} \overline{\tilde{k}_{b_{i}}^{\beta}\left(\overline{a_{m}}\right)}+\sum_{n=1}^{\infty} \frac{d_{n}}{1-\bar{b}_{i} b_{n}} \overline{\tilde{k}_{\bar{b}}^{\alpha}\left(a_{j}\right)}\right)$.

When $\left(l_{k}\right)_{k}$ is empty, we have

$s_{i, j}=\frac{1}{\beta^{\prime}\left(b_{i}\right)}\left(\sum_{m=1}^{\infty} \frac{c_{m} a_{m}}{1-\bar{a}_{j} a_{m}} \frac{\beta^{\#}\left(a_{m}\right)}{a_{m}-\bar{b}_{i}}+\sum_{n=1}^{\infty} \frac{d_{n}}{1-\bar{b}_{i} b_{n}} \frac{\alpha^{\#}\left(b_{n}\right)}{b_{n}-\bar{a}_{j}}\right)$.

When $\left(l_{k}\right)_{k}$ is not empty, we have

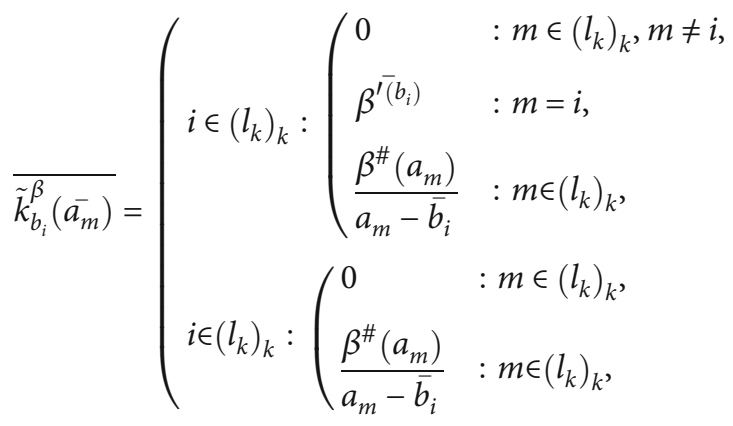

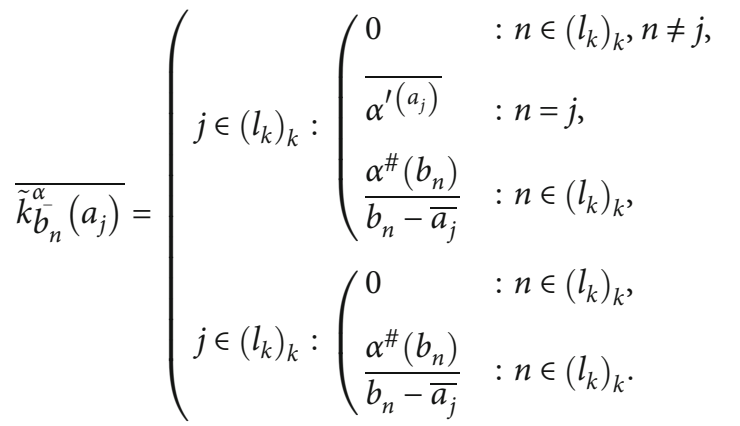

Therefore, we have 4 cases, $\left\{j \in\left(l_{k}\right)_{k}\right.$ and $\left.i \in\left(l_{k}\right)_{k}\right\},\{j \in$ $\left(l_{k}\right)_{k}$ and $\left.i \in\left(l_{k}\right)_{k}\right\}, \quad\left\{j \in\left(l_{k}\right)_{k}\right.$ and $\left.i \in\left(l_{k}\right)_{k}\right\}$, and $\left\{j \in\left(l_{k}\right)_{k}\right.$ andi $\epsilon$ $\left.\left(l_{k}\right)_{k}\right\}$. In all these cases, we decompose, add, and subtract as in the finite-dimensional case. Using the fact that $a_{1}=\overline{b_{1}}$, we obtain

$s_{i, j}=\frac{\bar{\beta}^{\prime}\left(b_{1}\right)\left(1-\overline{a_{j} b_{1}}\right) s_{1, j}-\bar{\beta}^{\prime}\left(b_{1}\right)\left(1-\overline{a_{1} b_{1}}\right) s_{1,1}+\bar{\beta}^{\prime}\left(b_{i}\right)\left(1-\overline{a_{1} b_{i}}\right) s_{i, 1}}{\beta^{\prime\left(b_{i}\right)}\left(1-\overline{a_{j} b_{i}}\right)}$.

Conversely, we proceed as in [9] for truncated Hankel operators but using the generalized characterization in Theorem 4.

As for Theorem 10, we also generalize the matrix representation of the ATHOs with respect to conjugate kernel bases.
Theorem 17. Let $B$ be a bounded linear transformation from $K_{\alpha}$ to $K_{\beta}$. We have $B \in \mathfrak{T}(\alpha, \beta)$ if and only if for any $i, j \geq 1$, we have

$$
p_{i, j}=\frac{\beta^{\prime}\left(b_{1}\right)\left(1-a_{j} b_{1}\right) p_{1, j}-\beta^{\prime}\left(b_{1}\right)\left(1-a_{1} b_{1}\right) p_{1,1}+\beta^{\prime}\left(b_{i}\right)\left(1-a_{1} b_{i}\right) p_{i, 1}}{\beta^{\prime}\left(b_{i}\right)\left(1-a_{j} b_{i}\right)} .
$$

Suppose that $\alpha$ and $\beta$ are inner functions such that $K_{\alpha}$ and $K_{\beta}$ have Clark bases $\left(v_{\eta_{j}}^{\alpha}\right)_{j \geq 1}$ and $\left(v_{\zeta_{i}}^{\beta}\right)_{i \geq 1}$, where $\left(\eta_{j}\right)_{j}$ and $\left(\zeta_{i}\right)_{i}$ are sequences of eigenvalues for some $U_{\lambda_{1}}^{\alpha}$ and some $U_{\lambda_{2}}^{\beta}$ satisfying the equations (30). We have the relations $\sqrt{\left|\alpha^{\prime}\left(\eta_{j}\right)\right|}=\left\|k_{\eta_{j}}^{\alpha}\right\|$ and $\sqrt{\left|\beta^{\prime}\left(\zeta_{i}\right)\right|}=\left\|k_{\zeta_{i}}^{\beta}\right\|$. Reorder these sequences such that $\eta_{l_{k}}=\overline{\zeta_{l}}$, for any $k \geq 1$ and that $1=l_{1} \epsilon$ $\left(l_{k}\right)_{k}$. We have the following theorem.

Theorem 18. Let $B$ be a bounded linear transformation from $K_{\alpha}$ to $K_{\beta} . B \in \mathfrak{H}(\alpha, \beta)$ if and only if

(1) When $\left(l_{k}\right)_{k \geq 1}$ is not empty, for $\left\{i \in\left(l_{k}\right)_{k}\right.$ and $\left.j \in\left(l_{k}\right)_{k}\right\}$ or $\left\{i \in\left(l_{k}\right)_{k}\right.$ and $j \in\left(l_{k}\right)_{k}$ and $\left.j \neq i\right\}$

$$
\begin{aligned}
t_{i, j}= & \frac{\sqrt{\left|\beta^{\prime}\left(\zeta_{1}\right)\right|}\left(\bar{\eta}_{j}-\zeta_{1}\right)}{\sqrt{\left|\beta^{\prime}\left(\zeta_{i}\right)\right|}\left(\bar{\eta}_{j}-\zeta_{i}\right)} t_{1, j} \\
& +\frac{\sqrt{\left|\alpha^{\prime}\left(\eta_{i}\right)\right|} \sqrt{\left|\beta^{\prime}\left(\zeta_{1}\right)\right|}\left(\bar{\eta}_{1}-\zeta_{j}\right)}{\sqrt{\left|\alpha^{\prime}\left(\eta_{j}\right)\right|} \sqrt{\left|\beta^{\prime}\left(\zeta_{i}\right)\right|}\left(\bar{\eta}_{j}-\zeta_{i}\right)} t_{1, i} .
\end{aligned}
$$

For $\left\{i \in\left(l_{k}\right)_{k}\right.$ and $\left.j \in\left(l_{k}\right)_{k}\right\}$ or $\left\{i \in\left(l_{k}\right)_{k}\right.$ and $\left.j \in\left(l_{k}\right)_{k}\right\}$,

$t_{i, j}=\frac{\sqrt{\left|\beta^{\prime}\left(\zeta_{1}\right)\right|}\left(\bar{\eta}_{j}-\zeta_{1}\right)}{\sqrt{\left|\beta^{\prime}\left(\zeta_{i}\right)\right|}\left(\bar{\eta}_{j}-\zeta_{i}\right)} t_{1, j}+\frac{\sqrt{\left|\alpha^{\prime}\left(\eta_{1}\right)\right|}\left(\bar{\eta}_{1}-\zeta_{j}\right)}{\sqrt{\left|\alpha^{\prime}\left(\eta_{j}\right)\right|}\left(\bar{\eta}_{j}-\zeta_{i}\right)} t_{i, 1}$.

(2) When $\left(l_{k}\right)_{k \geq 1}$ is empty, for any $i, j \geq 1$

$$
\begin{aligned}
t_{i, j}= & \frac{\sqrt{\left|\beta^{\prime}\left(\zeta_{1}\right)\right|}\left(\bar{\eta}_{j}-\zeta_{1}\right)}{\sqrt{\left|\beta^{\prime}\left(\zeta_{i}\right)\right|}\left(\bar{\eta}_{j}-\zeta_{i}\right)} t_{1, j} \\
& -\frac{\sqrt{\left|\alpha^{\prime}\left(\eta_{1}\right)\right|} \sqrt{\left|\beta^{\prime}\left(\zeta_{1}\right)\right|}\left(\bar{\eta}_{1}-\zeta_{1}\right)}{\sqrt{\left|\alpha^{\prime}\left(\eta_{j}\right)\right|} \sqrt{\left|\beta^{\prime}\left(\zeta_{i}\right)\right|}\left(\bar{\eta}_{j}-\zeta_{i}\right)} t_{1,1} \\
& +\frac{\sqrt{\left|\alpha^{\prime}\left(\eta_{1}\right)\right|}\left(\bar{\eta}_{1}-\zeta_{j}\right)}{\sqrt{\left|\alpha^{\prime}\left(\eta_{j}\right)\right|}\left(\bar{\eta}_{j}-\zeta_{i}\right)} t_{i, 1} .
\end{aligned}
$$


Proof. The proof is similar to the one of Theorem 14, but we use formula (44). For the converse implication, we proceed as in Theorem 21, except using the following characterization of ATHOs in Theorem 4, there exist $\chi \in K_{\alpha}$ and $\psi \in K_{\beta}$,

$$
B-\left(U_{\lambda_{2}}^{\beta}\right)^{*} B\left(U_{\lambda_{1}}^{\alpha}\right)^{*}=\psi \otimes k_{0}^{\alpha}+\tilde{k}_{0}^{\beta} \otimes \chi .
$$

As for Theorem 15, we deduce the matrix representation with respect to modified Clark bases.

Theorem 19. A bounded linear transformation $B$ from $K_{\alpha}$ to $K_{\beta}$ belongs to $\mathfrak{S}(\alpha, \beta)$ if and only if

(1) When $\left(l_{k}\right)_{k \geq 1}$ is not empty, for $\left\{i \in\left(l_{k}\right)_{k}\right.$ and $\left.j \in\left(l_{k}\right)_{k}\right\}$ or $\left\{i \in\left(l_{k}\right)_{k}\right.$ and $j \in\left(l_{k}\right)_{k}$ and $\left.j \neq i\right\}$

$$
\begin{aligned}
u_{i, j}= & \frac{\omega_{1}^{\beta}}{\omega_{i}^{\beta}} \frac{\sqrt{\left|\beta^{\prime}\left(\zeta_{1}\right)\right|}\left(\overline{\eta_{j}}-\zeta_{1}\right)}{\sqrt{\left|\beta^{\prime}\left(\zeta_{i}\right)\right|}\left(\overline{\eta_{j}}-\zeta_{i}\right)} u_{1, j} \\
& +\frac{\omega_{j}^{\alpha}}{\omega_{1}^{\alpha}} \frac{\sqrt{\left|\alpha^{\prime}\left(\eta_{i}\right)\right|} \sqrt{\left|\beta^{\prime}\left(\zeta_{1}\right)\right|}\left(\overline{\eta_{1}}-\zeta_{j}\right)}{\sqrt{\left|\alpha^{\prime}\left(\eta_{j}\right)\right|} \sqrt{\left|\beta^{\prime}\left(\zeta_{i}\right)\right|}\left(\overline{\eta_{j}}-\zeta_{i}\right)} u_{1, i} .
\end{aligned}
$$

For $\left\{i \in\left(l_{k}\right)_{k}\right.$ and $\left.j \in\left(l_{k}\right)_{k}\right\}$ or $\left\{i \in\left(l_{k}\right)_{k}\right.$ and $\left.j \in\left(l_{k}\right)_{k}\right\}$,

$$
\begin{aligned}
u_{i, j}= & \frac{\omega_{1}^{\beta}}{\omega_{i}^{\beta}} \frac{\sqrt{\left|\beta^{\prime}\left(\zeta_{1}\right)\right|}\left(\overline{\eta_{j}}-\zeta_{1}\right)}{\sqrt{\left|\beta^{\prime}\left(\zeta_{i}\right)\right|}\left(\overline{\eta_{j}}-\zeta_{i}\right)} u_{1, j} \\
& +\frac{\omega_{j}^{\alpha}}{\omega_{1}^{\alpha}} \frac{\sqrt{\left|\alpha^{\prime}\left(\eta_{1}\right)\right|}\left(\overline{\eta_{1}}-\zeta_{j}\right)}{\sqrt{\left|\alpha^{\prime}\left(\eta_{j}\right)\right|}\left(\overline{\eta_{j}}-\zeta_{i}\right)} u_{i, 1}
\end{aligned}
$$

(2) When $\left(l_{k}\right)_{k \geq 1}$ is empty

$$
\begin{aligned}
u_{i, j}= & \frac{\omega_{1}^{\beta}}{\omega_{i}^{\beta}} \frac{\sqrt{\left|\beta^{\prime}\left(\zeta_{1}\right)\right|}\left(\overline{\eta_{j}}-\zeta_{1}\right)}{\sqrt{\left|\beta^{\prime}\left(\zeta_{i}\right)\right|\left(\overline{\eta_{j}}-\zeta_{i}\right)} u_{1, j}} \\
& -\frac{\omega_{j}^{\alpha} \omega_{1}^{\beta}}{\omega_{1}^{\alpha} \omega_{i}^{\beta}} \frac{\sqrt{\left|\alpha^{\prime}\left(\eta_{1}\right)\right|} \sqrt{\left|\beta^{\prime}\left(\zeta_{1}\right)\right|}\left(\overline{\eta_{1}}-\zeta_{1}\right)}{\sqrt{\left|\alpha^{\prime}\left(\eta_{j}\right)\right|} \sqrt{\left|\beta^{\prime}\left(\zeta_{i}\right)\right|}\left(\overline{\eta_{j}}-\zeta_{i}\right)} u_{1,1} \\
& +\frac{\omega_{j}^{\alpha}}{\omega_{1}^{\alpha}} \frac{\sqrt{\left|\alpha^{\prime}\left(\eta_{1}\right)\right|}\left(\overline{\eta_{1}}-\zeta_{j}\right)}{\sqrt{\left|\alpha^{\prime}\left(\eta_{j}\right)\right|}\left(\overline{\eta_{j}}-\zeta_{i}\right)} u_{i, 1} .
\end{aligned}
$$

5.2. Matrix Representation of ATTOs. To get the matrix representation of ATTOs on interpolating model spaces, we will work as in the proof of Theorem 2.2 in [8]. We first need to explore the action of $V_{\xi, c}$ on $\mathfrak{T}(\alpha, \beta)$, where $V_{\xi, c}$ acts from $K_{\alpha}$ into $K_{\alpha \circ \tau_{\xi, c}}$ and is defined by $V_{\xi, c} f=\sqrt{\tau_{\xi, c}{ }^{\prime}}\left(f \circ \tau_{\xi, c}\right)$, $\tau_{\xi, c}(z)=\xi(c-z) /(1-\bar{c} z)$. ([13]).

Proposition 20. Let $\alpha$ and $\beta$ be two inner functions, $\varphi \in L^{2}$ and $A_{\varphi}^{\alpha, \beta} \in \mathfrak{I}(\alpha, \beta)$. Then for some $c \in \mathbb{D}$ and $\xi \in \mathbb{T}$,

$$
V_{\xi, c} A_{\varphi}^{\alpha, \beta} V_{\xi, c}^{-1}=A_{\varphi \circ \tau_{\xi, c}}^{\alpha \circ \tau_{\xi, c} \beta \circ \tau_{\xi, c}}
$$

Proof. For $f \in K_{\alpha}, \varphi \in L^{2}, \xi \in \mathbb{T}$ and $a \in \mathbb{D}$, we have

$$
\begin{aligned}
V_{\xi, c}\left[P_{\beta}(\varphi f)\right](z)= & \sqrt{\tau_{\xi, c}^{\prime}(z)}\left[P_{\beta}(\varphi f)\right] \circ \tau_{\xi, c}(z) \\
= & \sqrt{\tau_{\xi, c}^{\prime}(z)}\left\langle\varphi f, k_{\tau_{\xi, c}(z)}^{\beta}\right\rangle \\
= & \sqrt{\tau_{\xi, c}^{\prime}(z)} \int_{\mathbb{T}} \varphi(\chi) f(\chi) \\
& \cdot \frac{1-\beta\left(\tau_{\xi, c}(z)\right) \overline{\beta(\chi)}}{1-\tau_{\xi, c}(z) \bar{\chi}} d \chi .
\end{aligned}
$$
have

Let $\chi=\tau_{\xi, c}(\omega)$. Since $\tau_{\xi, c}^{\prime}(z)=\xi\left(|c|^{2}-1\right) /\left((1-\bar{c} z)^{2}\right)$, we

$$
\begin{aligned}
V_{\xi, c}\left[P_{\beta}(\varphi f)\right](z)= & \sqrt{\tau_{\xi, c}^{\prime}(z)} \int_{\mathbb{T}} \varphi\left(\tau_{\xi, c}(\omega)\right) f\left(\tau_{\xi, c}(\omega)\right) \\
& \cdot \frac{1-\beta\left(\tau_{\xi, c}(z)\right) \overline{\beta\left(\tau_{\xi, c}(\omega)\right.}}{1-\tau_{\xi, c}(z) \overline{\tau_{\xi, c}(\omega)}}\left|\tau_{\xi, c}^{\prime}(\omega)\right| d \omega \\
= & \sqrt{\tau_{\xi, c}^{\prime}(z)} \int_{\mathbb{T}} \varphi\left(\tau_{\xi, c}(\omega)\right) f\left(\tau_{\xi, c}(\omega)\right) \\
& \cdot \frac{1-z \bar{\omega}}{1-\tau_{\xi, c}(z) \overline{\tau_{\xi, c}(\omega)}} \overline{k_{z}^{\beta \circ \tau_{\xi, c}}(\omega)}\left|\tau_{\xi, c}^{\prime}(\omega)\right| d \omega .
\end{aligned}
$$

Since

$$
\sqrt{\tau_{\xi, c}^{\prime}(z)}\left|\tau_{\xi, c}^{\prime}(\omega)\right| \frac{1-z \bar{\omega}}{1-\tau_{\xi, c}^{\prime}(z) \overline{\tau_{\xi, c}^{\prime}(\omega)}}=\sqrt{\tau_{\xi, c}^{\prime}(\omega)}
$$

we have

$$
\begin{aligned}
V_{\xi, c}\left[P_{\beta}(\varphi f)\right](z)= & \int_{\mathbb{T}} \varphi\left(\tau_{\xi, c}(\omega)\right) f\left(\tau_{\xi, c}(\omega)\right) \\
& \cdot \sqrt{\tau_{\xi, c}^{\prime}}(\omega) \overline{k_{z}^{\beta \circ \tau_{\xi, c}}(\omega)} d \omega \\
= & \int_{\mathbb{T}} \varphi \circ \tau_{\xi, c}(\omega) V_{\xi, c} f(\omega) \overline{k_{z}^{\beta \circ \tau_{\xi, c}}(\omega)}(\omega) d \omega \\
= & \left\langle\varphi \circ \tau_{\xi, c} V_{\xi, c} f, k_{z}^{\beta \circ \tau_{\xi, c}}(\omega)\right\rangle \\
= & P_{\beta \circ \tau_{\xi, c}}\left(\varphi \circ \tau_{\xi, c} V_{\xi, c} f\right)(z) .
\end{aligned}
$$


In conclusion,

$$
V_{\xi, c} A_{\varphi}^{\alpha, \beta}=A_{\varphi \circ \tau_{\xi, c}^{\alpha}}^{\alpha \circ \tau_{\xi, c} \beta \circ \tau_{\xi, c}} V_{\xi, c}
$$

Applying $V_{\xi, c}^{-1}=V_{\xi, c}^{*}$, we get the result.

As before, the formula (20) is also true for ATTOs. We will prove that the matrix characterizations of ATTOs on finite-dimensional model space obtained in [6] are also true in the infinite case. Denote by $\left(a_{l_{k}}\right)_{k \geq 1}$ the subsequence of common zeros between $\alpha$ and $\beta$ ordered such that $a_{l_{k}}=b_{l_{k}}$, for any $k \geq 1$ and that $1=l_{1} \in\left(l_{k}\right)_{k}$ when $\left(l_{k}\right)_{k}$ is not empty.

Theorem 21. A bounded linear transformation $A$ from $K_{\alpha}$ to $K_{\beta}$ belongs to $\mathfrak{I}(\alpha, \beta)$ if and only if its matrix representation with respect to the kernel bases satisfies

(1) When $\left(l_{k}\right)_{k \geq 1}$ is not empty, for $\left\{i \in\left(l_{k}\right)_{k}\right.$ and $\left.j \in\left(l_{k}\right)_{k}\right\}$ or $\left\{i \in\left(l_{k}\right)_{k}\right.$ and $j \in\left(l_{k}\right)_{k}$ and $\left.j \neq i\right\}$

$$
s_{i, j}=\frac{\bar{\beta}^{\prime}\left(b_{1}\right)\left(\overline{a_{j}}-\overline{b_{1}}\right) s_{1, j}+\bar{\beta}^{\prime}\left(b_{1}\right)\left(\overline{a_{1}}-\overline{b_{i}}\right) s_{1, i}}{\bar{\beta}^{\prime}\left(b_{i}\right)\left(\overline{a_{j}}-\overline{b_{i}}\right)} .
$$

For $\left\{i \in\left(l_{k}\right)_{k}\right.$ and $\left.j \in\left(l_{k}\right)_{k}\right\}$ or $\left\{i \in\left(l_{k}\right)_{k}\right.$ and $\left.j \in\left(l_{k}\right)_{k}\right\}$,

$$
s_{i, j}=\frac{\bar{\beta}^{\prime}\left(b_{1}\right)\left(\overline{a_{j}}-\overline{b_{1}}\right) s_{1, j}+\bar{\beta}^{\prime}\left(b_{i}\right)\left(\overline{a_{1}}-\overline{b_{i}}\right) s_{i, 1}}{\bar{\beta}^{\prime}\left(b_{i}\right)\left(\overline{a_{j}}-\overline{b_{i}}\right)} .
$$

(2) When $\left(l_{k}\right)_{k \geq 1}$ is empty

$s_{i, j}=\frac{\bar{\beta}^{\prime}\left(b_{1}\right)\left(\overline{a_{j}}-\overline{b_{1}}\right) s_{1, j}-\bar{\beta}^{\prime}\left(b_{1}\right)\left(\overline{a_{1}}-\overline{b_{1}}\right) s_{1,1}+\bar{\beta}^{\prime}\left(b_{i}\right)\left(\overline{a_{1}}-\overline{b_{i}}\right) s_{i, 1}}{\bar{\beta}^{\prime}\left(b_{i}\right)\left(\overline{a_{j}}-\overline{b_{i}}\right)}$.
Proof. The proof of necessity is the same as in [6] for ATTOs acting on finite-dimensional model spaces. Conversely, consider any bounded linear transformation from $K_{\alpha}$ into $K_{\beta}$ whose matrix representation satisfies Theorem 21 . To show that $A \in \mathfrak{I}(\alpha, \beta)$, or equivalently to show that $A$ satisfies the characterization in Theorem 4 , we will find a $\chi \in K_{\alpha}$ and a $\psi \in K_{\beta}$ such that

$$
A-S_{\beta} A S_{\alpha}^{*}=\psi \otimes k_{0}^{\alpha}+k_{0}^{\beta} \otimes \chi .
$$

This is equivalent to

$$
\begin{aligned}
& \left\langle A k_{a_{j}}^{\alpha}, \tilde{k}_{b_{i}}^{\beta}\right\rangle-\left\langle S_{\beta} A S_{\alpha}^{*} k_{a_{j}}^{\alpha}, \tilde{k}_{b_{i}}^{\beta}\right\rangle \\
& =\left\langle\psi \otimes k_{0}^{\alpha}\left(k_{a_{j}}^{\alpha}\right), \tilde{k}_{b_{i}}^{\beta}\right\rangle+\left\langle k_{0}^{\beta} \otimes \chi\left(k_{a_{j}}^{\alpha}\right), \tilde{k}_{b_{i}}^{\beta}\right\rangle,
\end{aligned}
$$

for any $i, j \geq 1$. Using the relations in Lemma 3 , when $b_{i} \neq 0$, for any $i \geq 1$, we have for every $i, j \geq 1$

$$
\begin{gathered}
\left(1-\frac{\overline{a_{j}}}{\overline{b_{i}}}\right)\left\langle A k_{a_{j}}^{\alpha}, \tilde{k}_{b_{i}}^{\beta}\right\rangle+\frac{\overline{a_{j}}}{\overline{\overline{b_{i}}}}\left\langle k_{a_{j}}^{\alpha}, A^{*} \tilde{k}_{0}^{\beta}\right\rangle \\
=k_{a_{j}}^{\alpha}(0)\left\langle\psi, \tilde{k}_{b_{i}}^{\beta}\right\rangle+\overline{\tilde{k}_{b_{i}}^{\beta}(0)}\left\langle k_{a_{j}}^{\alpha}, \chi\right\rangle,
\end{gathered}
$$

or

$$
\left(1-\frac{\overline{a_{j}}}{\overline{b_{i}}}\right) \overline{\beta^{\prime}\left(b_{i}\right)} s_{i, j}+\frac{\overline{a_{j}}}{\overline{\overline{b_{i}}}}\left\langle k_{a_{j}}^{\alpha}, A^{*} \tilde{k}_{0}^{\beta}\right\rangle=\overline{\tilde{\psi}\left(b_{i}\right)}-\frac{\overline{\beta(0)}}{\overline{b_{i}}} \overline{\chi\left(a_{j}\right)},
$$

for any $i, j \geq 1$.

When $\left(l_{k}\right)_{k}$ is not empty, suppose that $\beta(0) \neq 0$. Since the matrix representation of $A$ satisfies the formulas in Theorem 21 , the above system is equivalent to

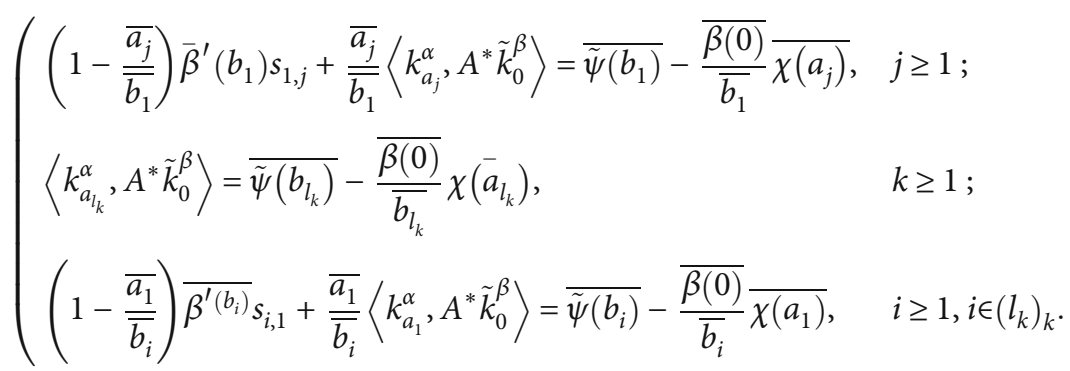


Set an arbitrary $\tilde{\psi}\left(b_{1}\right)$, then the solution of the system is

$$
\left(\begin{array}{ll}
\overline{\chi\left(a_{j}\right)}=\frac{\overline{b_{1}}}{\overline{\beta(0)}}\left(\overline{\tilde{\psi}\left(b_{1}\right)}-\left(1-\frac{\overline{a_{j}}}{\overline{b_{1}}}\right) \overline{\beta^{\prime}\left(b_{1}\right)} s_{1, j}-\frac{\overline{a_{j}}}{\overline{b_{1}}}\left\langle k_{a_{j}}^{\alpha}, A^{*} \tilde{k}_{0}^{\beta}\right\rangle\right), & j \geq 1 ; \\
\overline{\tilde{\psi}\left(b_{i}\right)}=-\left\langle k_{a_{i}}^{\alpha}, A^{*} \tilde{k}_{0}^{\beta}\right\rangle-\frac{\overline{\beta(0)}}{\overline{b_{i}}} \overline{\chi\left(a_{i}\right)} ; & i \in\left(l_{k}\right)_{k} . \\
\overline{\tilde{\psi}\left(b_{i}\right)}=\left(1-\frac{\overline{a_{1}}}{\overline{b_{i}}}\right) \overline{\beta^{\prime}\left(b_{i}\right)} s_{i_{1} 1}+\frac{\overline{a_{1}}}{\overline{b_{i}}}\left\langle k_{a_{1}}^{\alpha}, A^{*} \tilde{k}_{0}^{\beta}\right\rangle+\frac{\overline{\beta(0)}}{\overline{b_{i}}} \overline{\chi\left(a_{1}\right)} . & i \geq 1, i \in\left(l_{k}\right)_{k} .
\end{array}\right.
$$

To show that the solutions of the system $\chi$ and $\psi$ are in $K_{\alpha}$ and $K_{\beta}$, respectively, it suffices to prove that $\chi$ and $\psi$ are the unique solutions in $K_{\alpha}$ and $K_{\beta}$ of the interpolation problems corresponding to $\left(a_{m}\right)_{m \geq 1}$ and $\left(b_{n}\right)_{n \geq 1}$ (39). In fact, since $A^{*} \tilde{k}_{0}^{\beta}, A^{*} \tilde{k}_{b_{1}}^{\beta}\left(a_{j}\right), \widetilde{A k_{a_{1}}^{\alpha}} \in K_{\beta}$, and $\left(a_{j}\right)_{j \geq 1},\left(b_{i}\right)_{i \geq 1}$ are Blaschke sequences, we have

$$
\begin{aligned}
& \sum_{j=1}^{+\infty}\left|\chi\left(a_{j}\right)\right|^{2}\left(1-\left|a_{j}\right|^{2}\right)
\end{aligned}
$$

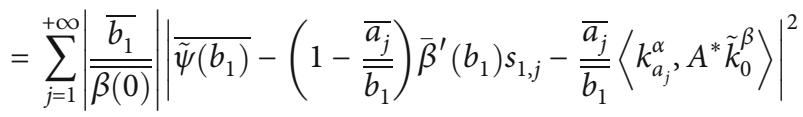

$$
\begin{aligned}
& \cdot\left(1-\left|a_{j}\right|^{2}\right) \leq C \sum_{j=1}^{+\infty}\left|A^{*} \tilde{k}_{b_{1}}^{\beta}\left(a_{j}\right)\right|^{2}\left(1-\left|a_{j}\right|^{2}\right)+C\left|\widetilde{\psi\left(b_{1}\right)}\right|^{2} \\
& \cdot \sum_{j=1}^{+\infty}\left(1-\left|a_{j}\right|^{2}\right)+C \sum_{j=1}^{+\infty}\left|A^{*} \tilde{k}_{0}^{\beta}\left(a_{j}\right)\right|^{2}\left(1-\left|a_{j}\right|^{2}\right)<\infty .
\end{aligned}
$$

$$
\begin{aligned}
& \sum_{i=1}^{+\infty}\left|\tilde{\psi}\left(b_{i}\right)\right|^{2}\left(1-\left|b_{i}\right|^{2}\right) \\
& =\sum_{i=1, i \in\left(l_{k}\right)_{k}}^{+\infty}\left|<k_{a_{j}}^{\alpha}, A^{*} \tilde{k}_{0}^{\beta}>+\frac{\overline{\beta(0)}}{\overline{b_{i}}} \overline{\chi\left(a_{i}\right)}\right|^{2}\left(1-\left|b_{i}\right|^{2}\right) \\
& +\sum_{i=1, i \in\left(l_{k}\right)_{k}}^{+\infty} \mid\left(1-\frac{\overline{a_{1}}}{\overline{b_{i}}}\right) \overline{\beta^{\prime}\left(b_{i}\right)} s_{i, 1}+\frac{\overline{a_{1}}}{\overline{b_{i}}} \\
& <k_{a_{1}}^{\alpha}, A^{*} \tilde{k}_{0}^{\beta}>+\left.\frac{\overline{\beta(0)}}{\overline{b_{i}}} \overline{\chi\left(a_{1}\right)}\right|^{2}\left(1-\left|b_{i}\right|^{2}\right) \\
& \leq C \sum_{i=1}^{+\infty}\left|A^{*} \tilde{k}_{0}^{\beta}\left(a_{i}\right)\right|^{2}\left(1-\left|a_{i}\right|^{2}\right)+C \sum_{i=1}^{+\infty}\left|\chi\left(a_{i}\right)\right|^{2} \\
& \cdot\left(1-\left|a_{i}\right|^{2}\right)+C \sum_{i=1}^{+\infty}\left|\widetilde{A k_{a_{1}}^{\alpha}}\left(b_{i}\right)\right|\left(1-\left|b_{i}\right|^{2}\right) \\
& +C\left(\left|\chi\left(a_{1}\right)\right|^{2}+\left|A^{*} \widetilde{k_{0}^{\beta}}\left(a_{1}\right)\right|^{2}\right) \sum_{i=1}^{+\infty}\left(1-\left|b_{i}\right|^{2}\right)<\infty .
\end{aligned}
$$

If $\beta(0)=0, \beta(c) \neq 0$ for some $c \in \mathbb{D}$. Let $\xi=1$, by Proposition $20, A \in \mathfrak{I}(\alpha, \beta)$ if and only if $V_{c} A V_{c}^{*} \in \mathfrak{I}\left(\alpha \circ \tau_{c}, \beta \circ \tau_{c}\right)$. Since $\beta \circ \tau_{c}(0)=\beta(c) \neq 0$, we need to show that the matrix representation of $V_{c} A V_{c}^{*},\left(w_{i, j}\right)$ with respect to the kernel bases $\left(k_{\tau_{c}\left(a_{m}\right)}^{\alpha \circ \tau_{c}}\right)_{m}$ and $\left(k_{\tau_{c}\left(b_{n}\right)}^{\beta \circ \tau_{c}}\right)_{n}$ satisfies the relations in Theorem 21. In fact, as in [8], we have

$$
\begin{aligned}
V_{c}^{*} k_{\tau_{c}\left(a_{j}\right)}^{\alpha \circ \tau_{c}}(z) & =i \frac{1-c \overline{a_{j}}}{\sqrt{1-|c|^{2}}} k_{a_{j}}^{\alpha}(z), V_{c}^{*} \tilde{k}_{\tau_{c}\left(b_{i}\right)}^{\beta \circ \tau_{c}}(z) \\
& =-i \frac{1-\bar{c} b_{i}}{\sqrt{1-|c|^{2}}} \tilde{k}_{b_{i}}^{\beta}(z), \\
w_{i, j}= & \frac{1}{\overline{\left(\beta \circ \tau_{c}\right)^{\prime}\left(b_{i}\right)}}\left\langle V_{c} A V_{c}^{*} k_{\tau_{c}\left(a_{j}\right)}^{\alpha o \tau_{c}}, \tilde{k}_{\tau_{c}\left(b_{i}\right)}^{\beta \circ \tau_{c}}\right\rangle \\
= & \frac{\frac{\left(\tau_{c}\right)^{\prime}\left(b_{i}\right)}{(\beta)^{\prime}\left(b_{i}\right)}}{\left(\frac{1-c \overline{a_{j}}}{\sqrt{1-|c|^{2}}}\right.} \bar{i} \frac{\overline{\left(1 \bar{c} b_{i}\right)}}{\sqrt{1-|c|^{2}}}\left\langle A k_{a_{j}}^{\alpha}, \tilde{k}_{b_{i}}^{\beta}\right\rangle \\
= & \frac{1-c \overline{a_{j}}}{1-c \overline{b_{i}}} s_{i, j},
\end{aligned}
$$

for any $i, j \geq 1$. We also have

$$
\begin{aligned}
\left(\beta \circ \tau_{c}\right)^{\prime}\left(\tau_{c}\left(b_{i}\right)\right) & =\frac{\beta^{\prime}\left(b_{i}\right)}{\tau_{c}^{\prime}\left(b_{i}\right)}, \tau_{c}(z)=\frac{c-z}{1-\bar{c} z} \text { and } \tau_{c}^{\prime}(z) \\
& =\frac{|c|^{2}-1}{(1-\bar{c} z)^{2}}
\end{aligned}
$$

using these formulas we can prove that every $w_{i, j}$ satisfies first relation in Theorem 21,

$$
\begin{aligned}
& \frac{\overline{\left(\beta \circ \tau_{c}\right)^{\prime\left(\tau_{c}\left(b_{1}\right)\right)}}\left(\overline{\tau_{c}\left(a_{j}\right)}-\overline{\tau_{c}\left(b_{1}\right)}\right) w_{1, j}+\overline{\left(\beta \circ \tau_{c}\right)^{\prime\left(\tau_{c}\left(b_{i}\right)\right)}}\left(\overline{\tau_{c}\left(a_{1}\right)}-\overline{\tau_{c}\left(b_{i}\right)}\right) w_{i, 1}}{\overline{\left(\beta \circ \tau_{c}\right)^{\prime\left(\tau_{c}\left(b_{i}\right)\right)}}\left(\overline{\tau_{c}\left(a_{j}\right)}-\overline{\tau_{c}\left(b_{i}\right)}\right)} \\
& =\frac{1-c \overline{a_{j}}}{1-c \bar{b}_{i}} s_{i, j}=w_{i, j} .
\end{aligned}
$$


If $\left(l_{k}\right)_{k}$ is empty, then the equation (70) becomes with the assumption $\beta(0) \neq 0$,

$$
\left(\begin{array}{l}
\left(1-\frac{\overline{a_{j}}}{\overline{b_{1}}}\right) \overline{\beta^{\prime}\left(b_{1}\right)} s_{1, j}+\frac{\overline{a_{j}}}{\overline{b_{1}}}\left\langle k_{a_{j}}^{\alpha}, A^{*} \tilde{k}_{0}^{\beta}\right\rangle=\overline{\tilde{\psi}\left(b_{1}\right)}-\frac{\overline{\beta(0)}}{\overline{b_{1}}} \overline{\chi\left(a_{j}\right)}, \quad j \geq 1 ; \\
\left(1-\frac{\overline{a_{1}}}{\overline{b_{i}}}\right) \overline{\beta^{\prime}\left(b_{i}\right)} s_{i, 1}+\frac{\overline{a_{1}}}{\overline{b_{i}}}\left\langle k_{a_{1}}^{\alpha}, A^{*} \tilde{k}_{0}^{\beta}\right\rangle=\overline{\tilde{\psi}\left(b_{i}\right)}-\frac{\overline{\beta(0)}}{\overline{b_{i}}} \overline{\chi\left(a_{1}\right)}, \quad i \geq 2 .
\end{array}\right.
$$

If we set an arbitrary $\tilde{\psi}\left(b_{1}\right)$, then, the solutions are

$$
\left(\begin{array}{ll}
\overline{\chi\left(a_{j}\right)}=\frac{\overline{b_{1}}}{\overline{\beta(0)}}\left(\overline{\tilde{\psi}\left(b_{1}\right)}-\left(1-\frac{\overline{a_{j}}}{\overline{b_{1}}}\right) \overline{\beta^{\prime}\left(b_{1}\right)} s_{1, j}-\frac{\overline{a_{j}}}{\overline{\overline{b_{1}}}}\left\langle k_{a_{j}}^{\alpha}, A^{*} \tilde{k}_{0}^{\beta}\right\rangle\right), & j \geq 1 ; \\
\overline{\tilde{\psi}\left(b_{i}\right)}=\left(1-\frac{\overline{a_{1}}}{\overline{b_{i}}}\right) \bar{\beta}^{\prime}\left(b_{i}\right) s_{i, 1}+\frac{\overline{a_{1}}}{\overline{b_{i}}}\left\langle k_{a_{1}}^{\alpha}, A^{*} \tilde{k}_{0}^{\beta}\right\rangle+\frac{\overline{\beta(0)}}{\overline{b_{i}}} \overline{\chi\left(a_{1}\right)}, \quad i \geq 2 .
\end{array}\right.
$$

It remains to check that $\chi \in K_{\alpha}$ and $\psi \in K_{\beta}$ by showing that they are solutions of the corresponding interpolation problems. The case $\beta(0)=0$ can be treated in the same way as in the previous case.

To get the matrix representation of ATTOs with respect to conjugate kernel bases $\left(\tilde{k}_{a_{m}}^{\alpha}\right)_{m}$ and $\left(\tilde{k}_{b_{n}}^{\beta}\right)_{n}$, we proceed as in Theorem 13 and use the fact that $C_{\beta} A C_{\alpha}=A_{\bar{\alpha} \beta \bar{\varphi}}^{\alpha, \beta}$ from the Theorem 5.

Theorem 22. A bounded linear transformation from $K_{\alpha}$ to $K_{\beta}$ belongs to $\mathfrak{I}(\alpha, \beta)$ if and only if

(1) When $\left(l_{k}\right)_{k \geq 1}$ is not empty, for $\left\{i \in\left(l_{k}\right)_{k}\right.$ and $\left.j \in\left(l_{k}\right)_{k}\right\}$ or $\left\{i \in\left(l_{k}\right)_{k}\right.$ and $j \in\left(l_{k}\right)_{k}$ and $\left.j \neq i\right\}$

$$
p_{i, j}=\frac{\beta^{\prime}\left(b_{1}\right)\left(a_{j}-b_{1}\right) p_{1, j}+\beta^{\prime}\left(b_{1}\right)\left(a_{1}-b_{i}\right) p_{1, i}}{\beta^{\prime}\left(b_{i}\right)\left(a_{j}-b_{i}\right)} .
$$

For $\left\{i \in\left(l_{k}\right)_{k}\right.$ and $\left.j \in\left(l_{k}\right)_{k}\right\}$ or $\left\{i \in\left(l_{k}\right)_{k}\right.$ and $\left.j \in\left(l_{k}\right)_{k}\right\}$,

$$
p_{i, j}=\frac{\beta^{\prime}\left(b_{1}\right)\left(a_{j}-b_{1}\right) p_{1, j}+\beta^{\prime}\left(b_{i}\right)\left(a_{1}-b_{i}\right) p_{i, 1}}{\beta^{\prime}\left(b_{i}\right)\left(a_{j}-b_{i}\right)} .
$$

(2) When $\left(l_{k}\right)_{k \geq 1}$ is empty

$p_{i, j}=\frac{\beta^{\prime}\left(b_{1}\right)\left(a_{j}-b_{1}\right) p_{1, j}-\beta^{\prime}\left(b_{1}\right)\left(a_{1}-b_{1}\right) p_{1,1}+\beta^{\prime}\left(b_{i}\right)\left(a_{1}-b_{i}\right) p_{i, 1}}{\beta^{\prime}\left(b_{i}\right)\left(a_{j}-b_{i}\right)}$.

Suppose that $\alpha$ and $\beta$ are inner functions such that the spaces $K_{\alpha}$ and $K_{\beta}$ have Clark bases, $\left(v_{\eta_{j}}^{\alpha}\right)_{j}$ and $\left(v_{\zeta_{i}}^{\beta}\right)_{i}$. Denote the subsequence of common elements of $\left(\eta_{j}\right)_{j}$ and $\left(\zeta_{i}\right)_{i}$ by $\left(\eta_{l_{k}}\right)_{k}=\left(\zeta_{l_{k}}\right)_{k}$ ordered such that $\eta_{l_{k}}=\zeta_{l_{k}}$, for any $k \geq 1$ and that $1=l_{1} \in\left(l_{k}\right)_{k}$.

Theorem 23. A bounded linear transformation belongs to $\mathfrak{T}(\alpha, \beta)$ if and only if

(1) When $\left(l_{k}\right)_{k \geq 1}$ is not empty, for $\left\{i \in\left(l_{k}\right)_{k}\right.$ and $\left.j \in\left(l_{k}\right)_{k}\right\}$ or $\left\{i \in\left(l_{k}\right)_{k}\right.$ and $j \in\left(l_{k}\right)_{k}$ and $\left.j \neq i\right\}$

$$
\begin{aligned}
t_{i, j}= & \frac{\sqrt{\left|\beta^{\prime}\left(\zeta_{1}\right)\right|}\left(1-\zeta_{1} \overline{\eta_{j}}\right)}{\sqrt{\left|\beta^{\prime}\left(\zeta_{i}\right)\right|}\left(1-\zeta_{i} \bar{\eta}_{j}\right)} t_{1, j} \\
& +\frac{\sqrt{\left|\alpha^{\prime}\left(\eta_{i}\right)\right|} \sqrt{\left|\beta^{\prime}\left(\zeta_{1}\right)\right|} \zeta_{1}\left(1-\zeta_{i} \bar{\eta}_{1}\right)}{\sqrt{\left|\alpha^{\prime}\left(\eta_{j}\right)\right|} \sqrt{\left|\beta^{\prime}\left(\zeta_{i}\right)\right|} \eta_{i}\left(1-\zeta_{i} \bar{\eta}_{j}\right)} t_{1, i}
\end{aligned}
$$

For $\left\{i \in\left(l_{k}\right)_{k}\right.$ and $\left.j \in\left(l_{k}\right)_{k}\right\}$ or $\left\{i \in\left(l_{k}\right)_{k}\right.$ and $\left.j \in\left(l_{k}\right)_{k}\right\}$,

$$
t_{i, j}=\frac{\sqrt{\left|\beta^{\prime}\left(\zeta_{1}\right)\right|}\left(1-\zeta_{1} \bar{\eta}_{j}\right)}{\sqrt{\left|\beta^{\prime}\left(\zeta_{i}\right)\right|}\left(1-\zeta_{i} \bar{\eta}_{j}\right)} t_{1, j}+\frac{\sqrt{\left|\alpha^{\prime}\left(\eta_{1}\right)\right|}\left(1-\zeta_{i} \bar{\eta}_{1}\right)}{\sqrt{\left|\alpha^{\prime}\left(\eta_{j}\right)\right|}\left(1-\zeta_{i} \bar{\eta}_{j}\right)} t_{i, 1} .
$$

(2) When $\left(l_{k}\right)_{k \geq 1}$ is empty

$$
\begin{aligned}
t_{i, j}= & \frac{\sqrt{\left|\beta^{\prime}\left(\zeta_{1}\right)\right|}\left(1-\zeta_{1} \bar{\eta}_{j}\right)}{\sqrt{\left|\beta^{\prime}\left(\zeta_{i}\right)\right|}\left(1-\zeta_{i} \overline{\eta_{j}}\right)} t_{1, j} \\
& -\frac{\sqrt{\left|\alpha^{\prime}\left(\eta_{1}\right)\right|} \sqrt{\left|\beta^{\prime}\left(\zeta_{1}\right)\right|}\left(1-\zeta_{1} \overline{\eta_{1}}\right)}{\sqrt{\left|\alpha^{\prime}\left(\eta_{j}\right)\right|} \sqrt{\left|\beta^{\prime}\left(\zeta_{i}\right)\right|}\left(1-\zeta_{i} \overline{\eta_{j}}\right)} t_{1,1} \\
& +\frac{\sqrt{\left|\alpha^{\prime}\left(\eta_{1}\right)\right|}\left(1-\zeta_{i} \bar{\eta}_{1}\right)}{\sqrt{\left|\alpha^{\prime}\left(\eta_{j}\right)\right|}\left(1-\zeta_{i} \bar{\eta}_{j}\right)} t_{i, 1} .
\end{aligned}
$$

Proof. The proof is the same as in Theorem 21, except using the equivalent characterization from Theorem 4 instead. ?

We also deduce the matrix representation with respect to the modified Clark bases.

Theorem 24. A bounded linear transformation belongs to $\mathfrak{I}(\alpha, \beta)$ if and only if 
(1) When $\left(l_{k}\right)_{k \geq 1}$ is not empty, for $\left\{i \in\left(l_{k}\right)_{k}\right.$ and $j \in\left(l_{k}\right)_{k}$ \}or $\left\{i \in\left(l_{k}\right)_{k}\right.$ and $j \in\left(l_{k}\right)_{k}$ and $\left.j \neq i\right\}$

$$
\begin{aligned}
u_{i, j}= & \frac{\omega_{1}^{\beta}}{\omega_{i}^{\beta}} \frac{\sqrt{\left|\beta^{\prime}\left(\zeta_{1}\right)\right|}\left(1-\zeta_{1} \overline{\eta_{j}}\right)}{\sqrt{\left|\beta^{\prime}\left(\zeta_{i}\right)\right|}\left(1-\zeta_{i} \overline{\eta_{j}}\right)} u_{1, j} \\
& +\frac{\omega_{j}^{\alpha}}{\omega_{1}^{\alpha}} \frac{\sqrt{\left|\alpha^{\prime}\left(\eta_{i}\right)\right|} \sqrt{\left|\beta^{\prime}\left(\zeta_{1}\right)\right|} \zeta_{1}\left(1-\zeta_{i} \overline{\eta_{1}}\right)}{\sqrt{\left|\alpha^{\prime}\left(\eta_{j}\right)\right|} \sqrt{\left|\beta^{\prime}\left(\zeta_{i}\right)\right|} \eta_{i}\left(1-\zeta_{i} \bar{\eta}_{j}\right)} u_{1, i} .
\end{aligned}
$$

$$
\begin{aligned}
& \text { For }\left\{i \in\left(l_{k}\right)_{k} \text { and } j \in\left(l_{k}\right)_{k}\right\} \text { or }\left\{i \in\left(l_{k}\right)_{k} \text { and } j \in\left(l_{k}\right)_{k}\right\} \\
& u_{i, j}=\frac{\omega_{1}^{\beta}}{\omega_{i}^{\beta}} \frac{\sqrt{\left|\beta^{\prime}\left(\zeta_{1}\right)\right|}\left(1-\zeta_{1} \bar{\eta}_{j}\right)}{\sqrt{\left|\beta^{\prime}\left(\zeta_{i}\right)\right|}\left(1-\zeta_{i} \bar{\eta}_{j}\right)} u_{1, j} \\
& +\frac{\omega_{j}^{\alpha}}{\omega_{1}^{\alpha}} \frac{\sqrt{\left|\alpha^{\prime}\left(\eta_{1}\right)\right|}\left(1-\zeta_{i} \overline{\eta_{1}}\right)}{\sqrt{\left|\alpha^{\prime}\left(\eta_{j}\right)\right|}\left(1-\zeta_{i} \overline{\eta_{j}}\right)} u_{i, 1} .
\end{aligned}
$$

(2) When $\left(l_{k}\right)_{k \geq 1}$ is empty

$$
\begin{aligned}
u_{i, j}= & \frac{\omega_{1}^{\beta}}{\omega_{i}^{\beta}} \frac{\sqrt{\left|\beta^{\prime}\left(\zeta_{1}\right)\right|}\left(1-\zeta_{1} \bar{\eta}_{j}\right)}{\sqrt{\left|\beta^{\prime}\left(\zeta_{i}\right)\right|}\left(1-\zeta_{i} \bar{\eta}_{j}\right)} u_{1, j} \\
& -\frac{\omega_{j}^{\alpha}}{\omega_{i}^{\beta}} \frac{\omega_{1}^{\beta}}{\omega_{1}^{\alpha}} \frac{\sqrt{\left|\alpha^{\prime}\left(\eta_{1}\right)\right|} \sqrt{\left|\beta^{\prime}\left(\zeta_{1}\right)\right|}\left(1-\zeta_{1} \overline{\eta_{1}}\right)}{\sqrt{\left|\alpha^{\prime}\left(\eta_{j}\right)\right|} \sqrt{\left|\beta^{\prime}\left(\zeta_{i}\right)\right|}\left(1-\zeta_{i} \bar{\eta}_{j}\right)} u_{1,1} \\
& +\frac{\omega_{j}^{\alpha}}{\omega_{1}^{\alpha}} \frac{\sqrt{\left|\alpha^{\prime}\left(\eta_{1}\right)\right|}\left(1-\zeta_{i} \overline{\eta_{1}}\right)}{\sqrt{\left|\alpha^{\prime}\left(\eta_{j}\right)\right|}\left(1-\zeta_{i} \bar{\eta}_{j}\right)} u_{i, 1} .
\end{aligned}
$$

Remark 25. Note that since $J^{\#} k_{a_{j}}^{\alpha}=k_{\overline{a_{j}}}^{\alpha^{\#}}$ and $J^{\#} \tilde{k}_{a_{j}}^{\alpha}=\tilde{k}_{\overline{a_{j}}}^{\alpha^{\#}}$, we have

$$
\begin{aligned}
s_{i, j} & =\frac{1}{\overline{\beta^{\prime}\left(b_{i}\right)}}\left\langle B k_{a_{j}}^{\alpha}, \tilde{k}_{b_{i}}^{\beta}\right\rangle=\frac{1}{\overline{\beta^{\prime}\left(b_{i}\right)}} \overline{\left\langle C_{\beta} B J^{\#} k_{\overline{a_{j}}}^{\alpha \#}, k_{b_{i}}^{\beta}\right\rangle} \\
& =\frac{1}{\overline{\beta^{\prime}\left(b_{i}\right)}}\left\langle J^{\#} B C_{\alpha} \tilde{k}_{a_{j}}^{\alpha}, \tilde{k}_{\overline{b_{i}}}^{\beta^{\#}}\right\rangle,
\end{aligned}
$$

and since $C_{\beta} B J^{\#}$ and $J^{\#} B C_{\alpha}$ are ATTOs by Theorem 4 ([10]), then we can deduce the matrix representation of an ATTO with respect to kernel and conjugate kernel bases, and to conjugate kernel and kernel bases in both finite and infinite dimensional cases, which matches with the work done in [14].
Similarly, we also can obtain the matrix representation of an ATHO with respect to kernel and conjugate kernel bases, and to conjugate kernel and kernel bases via the passage formulas.

\section{Data Availability}

There is no underlying data in this paper, and all of its research is the derivation of basic theory.

\section{Conflicts of Interest}

The authors declare that they have no conflicts of interest.

\section{Acknowledgments}

We thank the referee for their time and comments. This research is supported by NSFC (nos. 12031002, 11901269, and LQ2019017).

\section{References}

[1] M. C. Camara and J. R. Partington, "Asymmetric truncated Toeplitz operators and Toeplitz operators with matrix symbol," Journal of Operator Theory, vol. 77, no. 2, pp. 455-479, 2017.

[2] B. Lanucha and M. Michalska, "When is an asymmetric truncated Hankel opertor equal to the zero operator?," Bulletin de la Société des sciences et des lettres de tódź, Série: Recherches sur les déformations, vol. 67, no. 3, pp. 69-76, 2017.

[3] D. Sarason, "Algebraic properties of truncated Toeplitz operators," Operators and Matrices, vol. 1, no. 4, pp. 491526, 2007.

[4] C. X. Gu, "Algebraic properties of truncated Hankel operators," preprint.

[5] B. Lanucha, "Asymmetric truncated Toeplitz operators of rank one," Computational Methods and Function Theory, vol. 18, no. 2, pp. 259-267, 2018.

[6] J. Jurasik and B. Lanucha, "Asymmetric truncated Toeplitz operators on finite-dimensional model spaces, in press," http://arxiv.org/abs/1611.00813v1.

[7] J. A. Cima, W. T. Ross, and W. R. Wogen, "Truncated Toeplitz operators on finite dimensional spaces," Operators and Matrices, vol. 2, no. 3, pp. 357-369, 2007.

[8] B. Lanucha, "Matrix representations of truncated Toeplitz operators," Journal of Mathematical Analysis and Applications, vol. 413, no. 1, pp. 430-437, 2014.

[9] B. Lanucha and M. Michalska, "Truncated Hankel operators and their matrices," Bulletin of the Korean Mathematical Society, vol. 56, no. 1, pp. 187-200, 2019.

[10] C. Gu, B. Łanucha, and M. Michalska, "Characterizations of asymmetric truncated Toeplitz and Hankel operators," Complex Anal. Oper. Theory, vol. 13, no. 3, pp. 673-684, 2019.

[11] D. N. Clark, "One dimensional perturbations of restricted shifts," Journal d'Analyse Mathématique, vol. 25, pp. 169191, 1972.

[12] J. Jurasik and B. Lanucha, "Asymmetric truncated Toeplitz operators equal to the zero operator," Annales Universitatis Mariae Curie-Sklodowska, sectio A-Mathematica, vol. 70, no. 2 , p. $51,2016$. 
[13] J. A. Cima, S. R. Garcia, W. T. Ross, and W. R. Wogen, “Truncated Toeplitz operators: spatial isomorphism, unitary equivalence, and similarity," Indiana University Mathematics Journal, vol. 59, no. 2, pp. 595-620, 2010.

[14] J. Jurasik and B. Lanucha, "Matrix representations of asymmetric truncated Toeplitz operators," Bulletin of the Malaysian Mathematical Sciences Society, vol. 44, no. 3, pp. 1443-1458, 2021. 